\title{
Airframe Noise Prediction of a Full Aircraft in Model and Full Scale Using a Lattice Boltzmann Approach
}

\author{
Ehab Fares, ${ }^{*}$ Benjamin Duda, $\uparrow$ \\ Exa GmbH, Curiestrasse 4, D-70563 Stuttgart, Germany \\ and \\ Mehdi R. Khorrami \\ NASA Langley Research Center, Hampton, Virginia, 23681
}

Unsteady flow computations are presented for a Gulfstream aircraft model in landing configuration, i.e., flap deflected $39^{\circ}$ and main landing gear deployed. The simulations employ the lattice Boltzmann solver PowerFLOW ${ }^{\circledR}$ to simultaneously capture the flow physics and acoustics in the near field. Sound propagation to the far field is obtained using a Ffowcs Williams and Hawkings acoustic analogy approach. Two geometry representations of the same aircraft are analyzed: an $18 \%$ scale, high-fidelity, semi-span model at wind tunnel Reynolds number and a full-scale, full-span model at half-flight Reynolds number. Previously published and newly generated model-scale results are presented; all full-scale data are disclosed here for the first time. Reynolds number and geometrical fidelity effects are carefully examined to discern aerodynamic and aeroacoustic trends with a special focus on the scaling of surface pressure fluctuations and farfield noise. An additional study of the effects of geometrical detail on farfield noise is also documented. The present investigation reveals that, overall, the model-scale and full-scale aeroacoustic results compare rather well. Nevertheless, the study also highlights that finer geometrical details that are typically not captured at model scales can have a non-negligible contribution to the farfield noise signature.

\section{Introduction}

Airframe noise constitutes a major component of the total noise generated by contemporary civil aircraft, especially during landing when the engines are typically at low thrust settings. The continuous growth in air travel has led to an increase in community noise exposure, resulting in stricter airport regulations nationally and in some cases also regionally. Expected future noise restrictions will inevitably lead to the development of overall quieter aircraft designs. A considerable number of studies, both experimental and numerical, have been conducted during the past two decades ${ }^{1}$ to acquire a better understanding of the sources of airframe noise, and to develop and advance noise reduction technologies. Flight tests and wind tunnel experiments targeting airframe noise sources have been instrumental in the development of component-level semi-empirical prediction tools ${ }^{2,3,4}$ such as NASA's Aircraft Noise Prediction Program (ANOPP). State-of-the-art numerical simulations addressing the combined flow and acoustic problems of isolated components using Computational Fluid Dynamics (CFD) and Computational Aeroacoustics (CAA) are summarized in various contributions to the workshop on Benchmark Problems for Airframe Noise Computations (BANC) and associated publications. ${ }^{5,6}$

Numerical simulations of the unsteady flow around an $18 \%$ scale, high-fidelity, semi-span model of a Gulfstream aircraft in landing configuration (flap deflected $39^{\circ}$ and main landing gear deployed) were recently performed using a Detached Eddy Simulation (DES) approach within NASA's FUN3D unstructured compressible Navier-Stokes solver $^{7}$ and a Lattice Boltzmann-Very Large Eddy Simulation (LB-VLES) approach within Exa Corporation's PowerFLOW ${ }^{\circledR}$ solver. ${ }^{8}$ The simulations, which combined multiple components of a complete aircraft including main landing gear interaction with the flap side edge of a high-lift system, compared well with measured quantities (steady and unsteady surface pressures and far field noise), demonstrating the general feasibility of both flow solvers to accurately simulate complex aeroacoustic interactions. The lattice Boltzmann algorithm provides a unique set of advantages in terms of computational efficiency, high frequency resolution and handling of very complex geometrical details, thus making it an ideal tool for detailed analysis of noise generation mechanisms ${ }^{9}$ and interaction effects. ${ }^{10}$

\footnotetext{
* Senior Technical Director, Aerospace Applications, Senior Member AIAA

$\uparrow$ Senior Application Engineer, Aerospace Aeroacoustics, Member AIAA

+ Aerospace Engineer, Computational AeroSciences Branch, Associate Fellow AIAA
} 
These advantages translate into an increased ability to simulate various noise reduction technologies and their effect on the aeroacoustic characteristics of isolated components and complete aircraft configurations. ${ }^{11}$ The accuracy of the numerical simulations was confirmed through comparisons with measurements obtained in the NASA Langley Research Center (LaRC) 14- by 22-Foot Subsonic Tunnel. ${ }^{10-15}$

The computational results presented in this paper extend the numerical simulations from the model scale wind tunnel geometry to the full-scale, full-span model of the aircraft in landing configuration to address two main objectives. The first objective is to investigate potential scale or Reynolds number (Re) effects on both aerodynamics and acoustics. To examine these effects, the simulations were performed at the same Mach number (M) but at two distinct Re. The second objective is to assess geometrical effects such as half-span vs. full-span, increased complexity of geometrical details, and their impact on farfield noise signature.

\section{Model Scale and Full Scale Geometries}

The $18 \%$ scale model is a high-fidelity, semi-span reproduction of a Gulfstream business jet. The geometry comprises a fuselage, wing, retractable flap, flow-through nacelle, pylon, and removable main landing gear. The semispan model was prepared with a fuselage stand-off at the symmetry plane to reduce tunnel floor boundary layer effects. A detailed description of this model is provided in Ref. 12. The full-scale geometry used for the present work was developed using a full-scale version of the $18 \%$ scale model fuselage, wing, and flow through nacelle; the flaps, including their complex bracket/track system, as well as the vertical and horizontal tails, were developed using asflown, full-scale geometry files furnished by the Gulfstream Aerospace Company (GAC). The main landing gear, including the wheel cavity, was developed from the original full-scale geometry files containing the finer details of the gear as deployed on the actual aircraft. The larger structures residing within the wheel well that could potentially alter the cavity volume were also included. Since the gear cavity extends across the fuselage connecting the two wheel wells, the full aircraft span had to be considered in the simulations. The model- and full-scale geometries are displayed in Fig. 1. In addition to the wheel well, the geometries are different at the flap side edge regions, main landing gear (MLG), and flap bracket region, as depicted in Fig. 2.

For both model-scale and full-scale simulations, $\mathrm{M}$ was chosen to be 0.2 . The Re based on mean aerodynamic chord was 3.4 million for the model scale and 10.5 million for the full-scale. The latter number is approximately half of the actual flight value of $\sim 21$ million for the aircraft at landing speeds. This Re, chosen to avoid the large computational cost and effort associated with resolution of thinner boundary and shear layers, is sufficiently high to capture the main scaling effects.

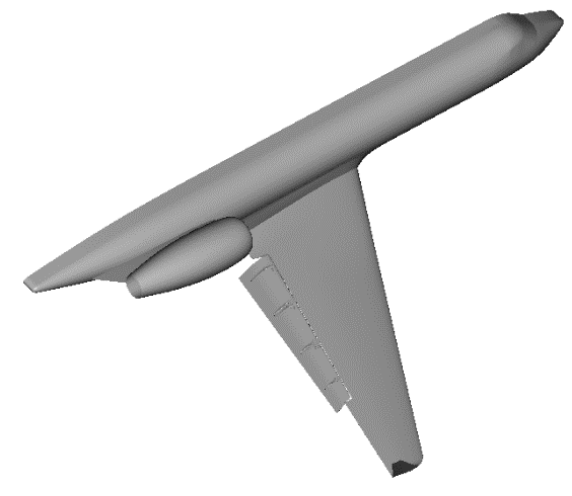

a) Semi-span, $18 \%$ scale model

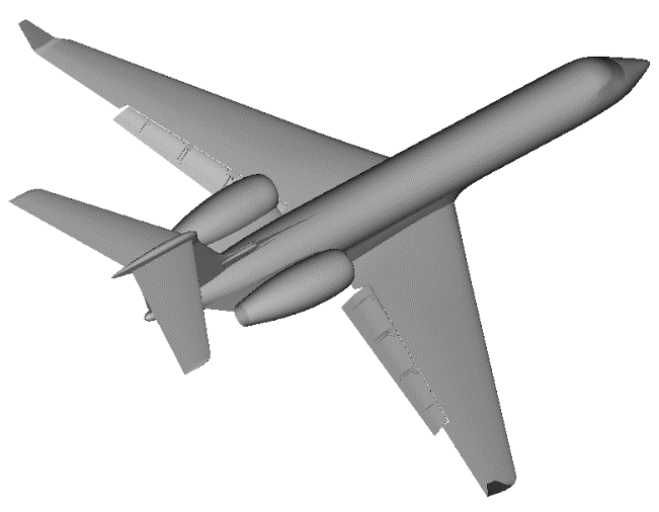

b) Full-scale, full-span model

Figure 1. Simulated aircraft geometries. 


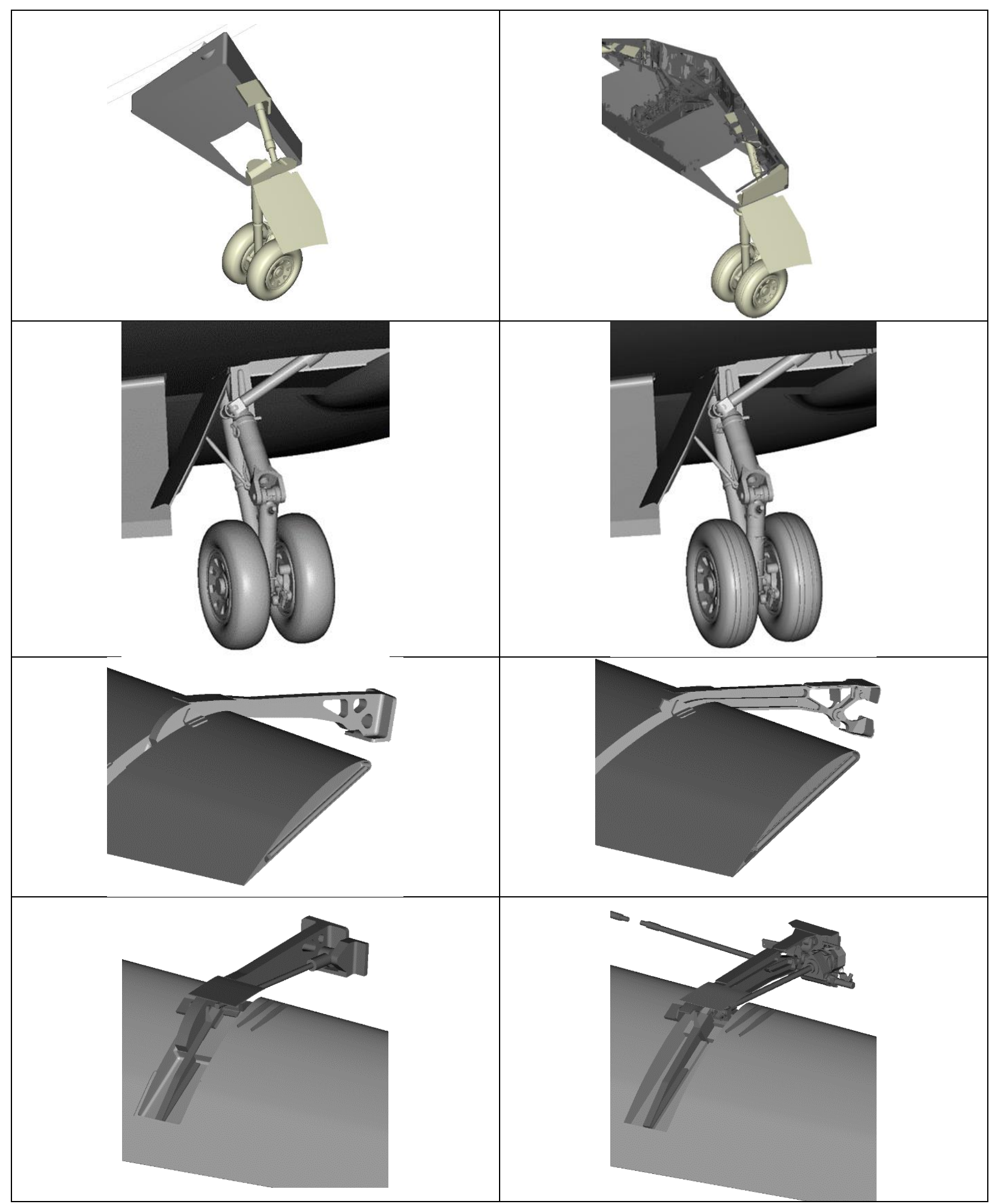

Figure 2. Comparison of geometrical details between model-scale (left) and full-scale (right) configurations. 


\section{Computational Approach}

The numerical simulations were performed using PowerFLOW ${ }^{\circledR}$, which is a compressible flow solver based on the three dimensional 19 state (D3Q19) lattice Boltzmann model (LBM). LBM is a CFD technology developed over the last 30 years ${ }^{16-20}$ and has been extensively validated for a wide variety of applications ranging from academic direct numerical simulation (DNS) cases to industrial flow problems in the fields of aerodynamics ${ }^{21}$ and aeroacoustics. ${ }^{8,22,23}$ In contrast to methods based on the Navier-Stokes (N-S) equations, LBM uses a simpler and more general physics formulation at the microscopic level. ${ }^{16}$ The LBM equations recover the macroscopic hydrodynamics of the NavierStokes equations ${ }^{21,24,25}$ through the Chapman-Enskog expansion. The local formulation of the LBM equations allows a highly efficient implementation for distributed computations on thousands of processors. The low dissipation and dispersion properties of the numerical scheme typically produce aerodynamic and aeroacoustic results that are comparable to those obtained with classical CFD solvers that use higher-order large eddy simulation (LES), as shown theoretically in Refs. 26 and 27, and demonstrated in the comparative study of flow over tandem cylinders presented in Ref. 6.

The classical LBM scheme is typically valid in the low Mach number regime up to M of 0.4. This method was applied for the work presented here. Recent extensions to the scheme ${ }^{28-30}$, however, allow the simulation of flows at higher Mach numbers as well.

\section{A. Turbulence Modelling}

The lattice Boltzmann flow simulation is equivalent to a Direct Numerical Simulation (DNS) of the flow. For high Re flows, such as those addressed in this work, the Lattice Boltzmann Very Large Eddy Simulation (LB-VLES) approach described in Refs. 18, 19, and 31 is used. The approach has been applied previously in Refs. 8, 9, and 10.

\section{B. Wall Treatment}

The standard Lattice-Boltzmann bounce-back boundary condition for no-slip or the specular reflection for freeslip condition are generalized through a volumetric formulation ${ }^{16,17}$ near the wall for arbitrarily oriented surface elements (surfels) within the Cartesian volume elements (voxels). This formulation of the boundary condition on a curved surface cutting the Cartesian grid is automatically mass, momentum, and energy conservative while maintaining the general spatial second-order accuracy of the underlying LBM numerical scheme. To reduce the resolution requirements near the wall for high Re flows, a hybrid wall function is used to model the region of the boundary layer closest to the solid surfaces. ${ }^{21,32}$

\section{Complex Geometry Handling and Meshing}

The lattice Boltzmann approach is solved on Cartesian meshes, which are generated automatically for any geometrically complex shape. Variable refinement regions (VR) can be defined to allow for local mesh refinement of the grid size by successive factors of two. The position and size of the local refinement regions used here correspond to the baseline configuration documented in Ref. 8. Said reference indicates that a medium resolution strategy is sufficient to capture both aerodynamics and acoustics up to the grid cut-off frequency and hence will be used here for all the comparisons. An illustration of the level of geometrical detail resolved in the simulation is given in Fig. 3 .

\section{Computational Procedure}

Initial and boundary conditions, as well as spatial and temporal resolution, are all identical to the previously published baseline configuration simulation. ${ }^{8}$ An acoustic analogy approach based on the Ffowcs Williams and Hawkings (FWH) formulation ${ }^{33}$ was used to propagate the computed near-field fluctuations to the farfield. The employed FWH formulation was based on the retarded-time formulation 1A by Farassat, ${ }^{34}$ extended to account for uniform mean flow convection effects to simulate the noise generated and measured in an ideal infinite wind tunnel. ${ }^{35}$ Integration was performed using solid surfaces of the aircraft and permeable surfaces surrounding different components of the aircraft. As shown in Ref. 8, both solid and permeable integrations including the complete aircraft produce the same farfield signature up to the cut-off frequency of the permeable surface, which is typically considerably lower than that for the solid surface due to the coarser volumetric mesh in the off-body region. In this paper, only the FWH propagation based on the solid formulation is reported. 

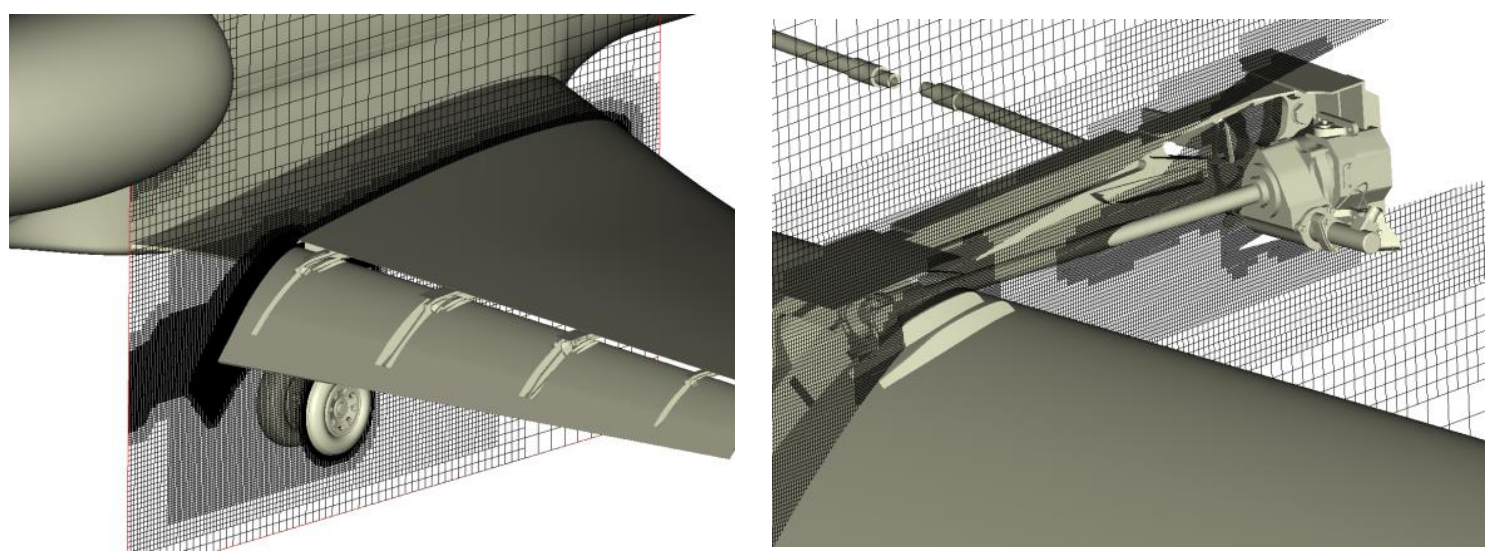

Figure 3. Full scale geometry and mesh at the inboard flap side edge (left) and flap middle bracket regions (right).

Every second mesh cell is shown.

\section{Results and Discussion}

\section{A. Global View}

Using a Gulfstream aircraft as the test bed, acoustic measurements during the 2006 NASA-Gulfstream joint flight test $^{36}$ highlighted the flap tips and main landing gear as the more prominent airframe noise sources for landing configuration. Accordingly, the current investigations are focused on these two important components.

The computational analysis presented here is based on three complex simulations: a) $18 \%$ model scale geometry at a Re of 3.4 million, b) $18 \%$ model scale geometry at a Re of 10.5 million, and c) full-scale geometry at Re 10.5 million. Comparative analyses of the first two simulations promote understanding of Re effects on the same geometry, while consideration of the latter two highlights geometrical effects. The first simulation represents the open-jet $14 \times 22$ tunnel (i.e., viscous flow on the floor and free-air in all directions above the floor), maintaining the same test conditions and model landing configurations as in the $14 \times 22$ tunnel setup. ${ }^{12-15}$ Results from this simulation, which have been extensively validated in previous studies, ${ }^{8,10,11}$ produced very good agreement for aerodynamic forces, pressure distributions, surface pressure fluctuations, flow field, and farfield noise. All the simulations were performed at an angle of attack of $3^{\circ}$ and $\mathrm{M}$ of 0.2 .

\section{B. Reynolds Number Effects}

As the most challenging configuration, the present comparison uses the $18 \%$ model scale semi-span geometry with deflected flap at $39^{\circ}$ and MLG deployed at two different Re. The grid resolution is adapted globally to enable the same resolution for the boundary and shear layers at the increased Re while maintaining the same mesh refinement strategy as depicted in Fig. 4. This approach nearly doubled the number of volume and surface elements required for the higher Re simulation.

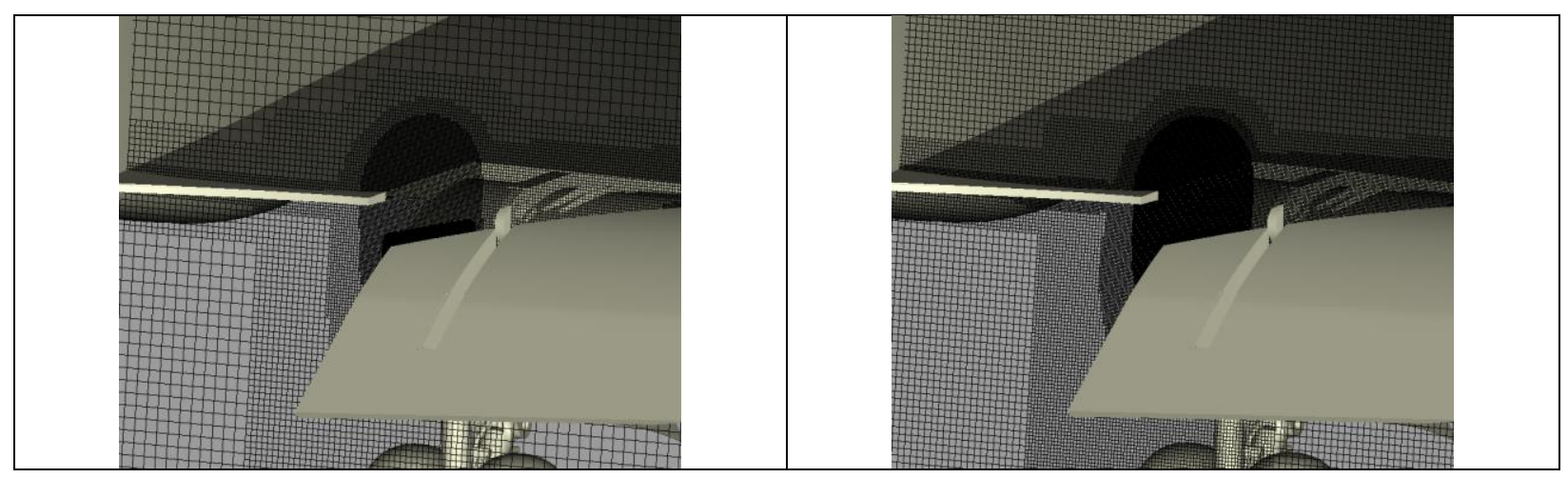

Figure 4. Comparison of mesh near the inboard flap side edge for the low Re (left) and the high Re (right) simulations. Every second mesh cell is shown. 
In general, both model scale simulations produced very similar flow patterns for wake separation at the flap brackets and formation of flap tip vortices, as can be seen globally in Fig. 5 (a, b) and Fig. 6 (a, b) and in more detail in Fig. 7 (a, b) and Fig. $8(a, b)$. Only minute differences in vortex roll-up and pressure imprint on the surface at the outboard flap tip region can be observed when comparing Figs. 5a and 5b, and 8a and 8b, respectively.

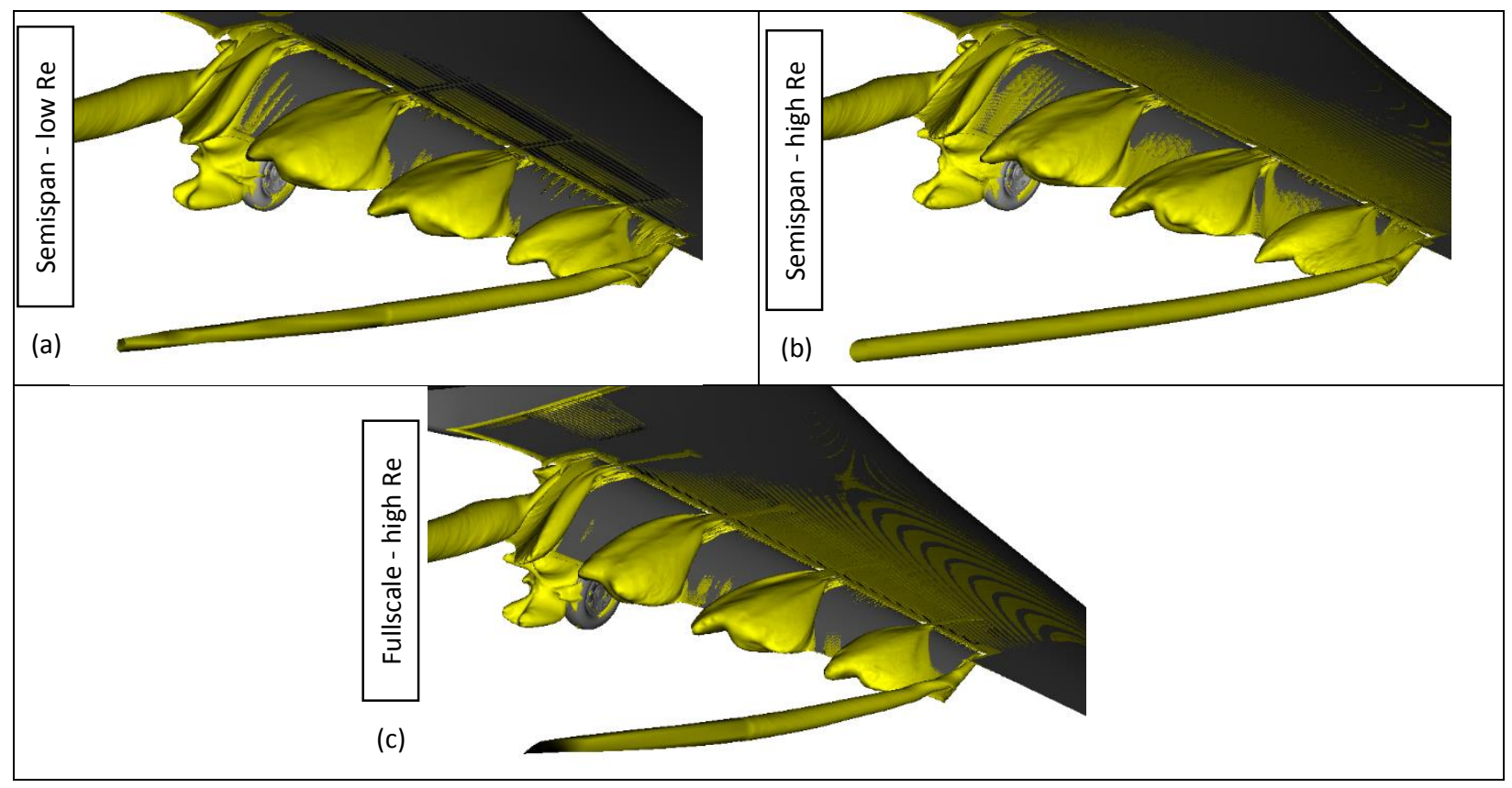

Figure 5. Comparison of isosurfaces of total pressure in the flap wake region.

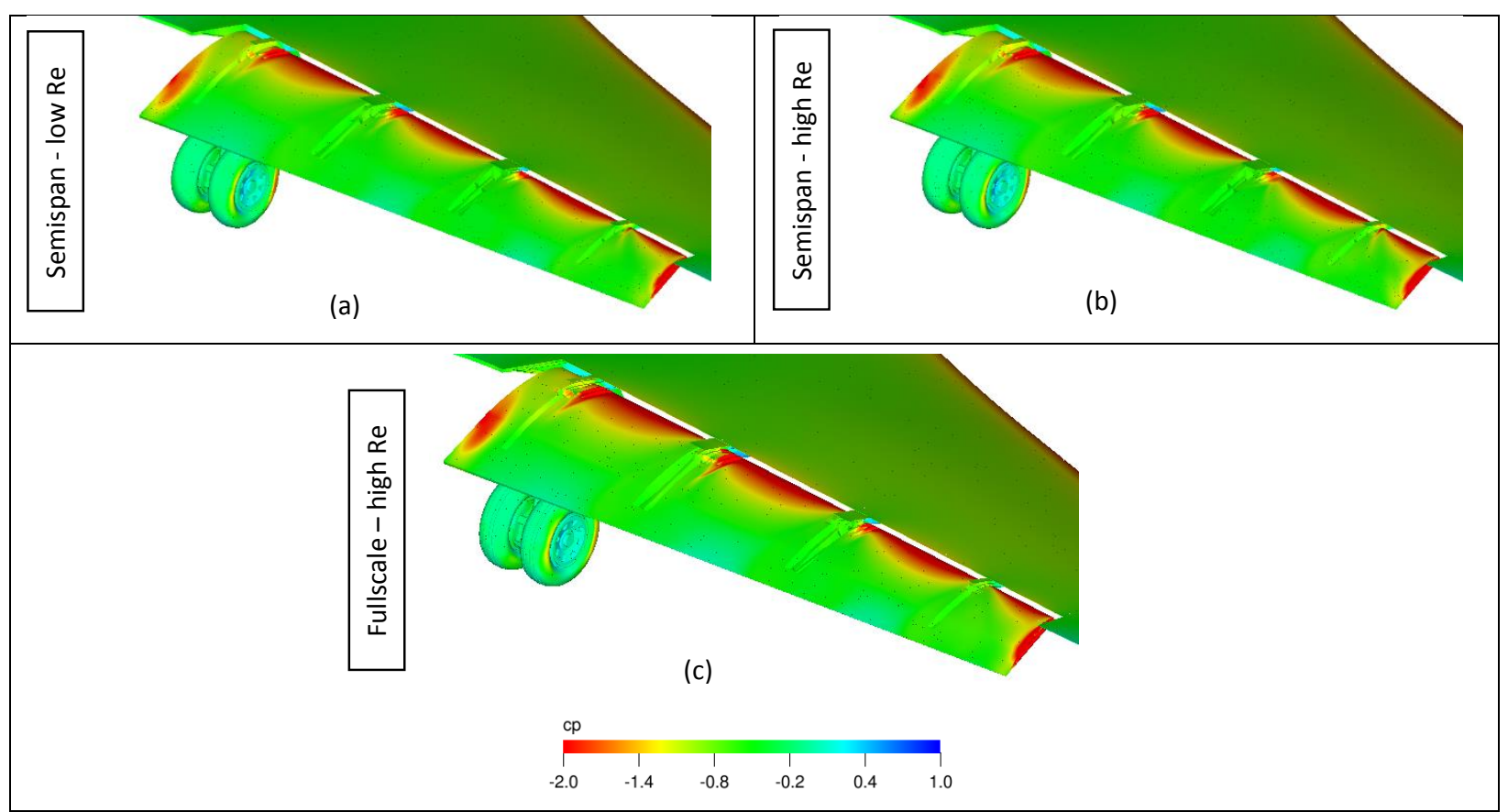

Figure 6. Comparison of static surface pressure distribution on the flap. 


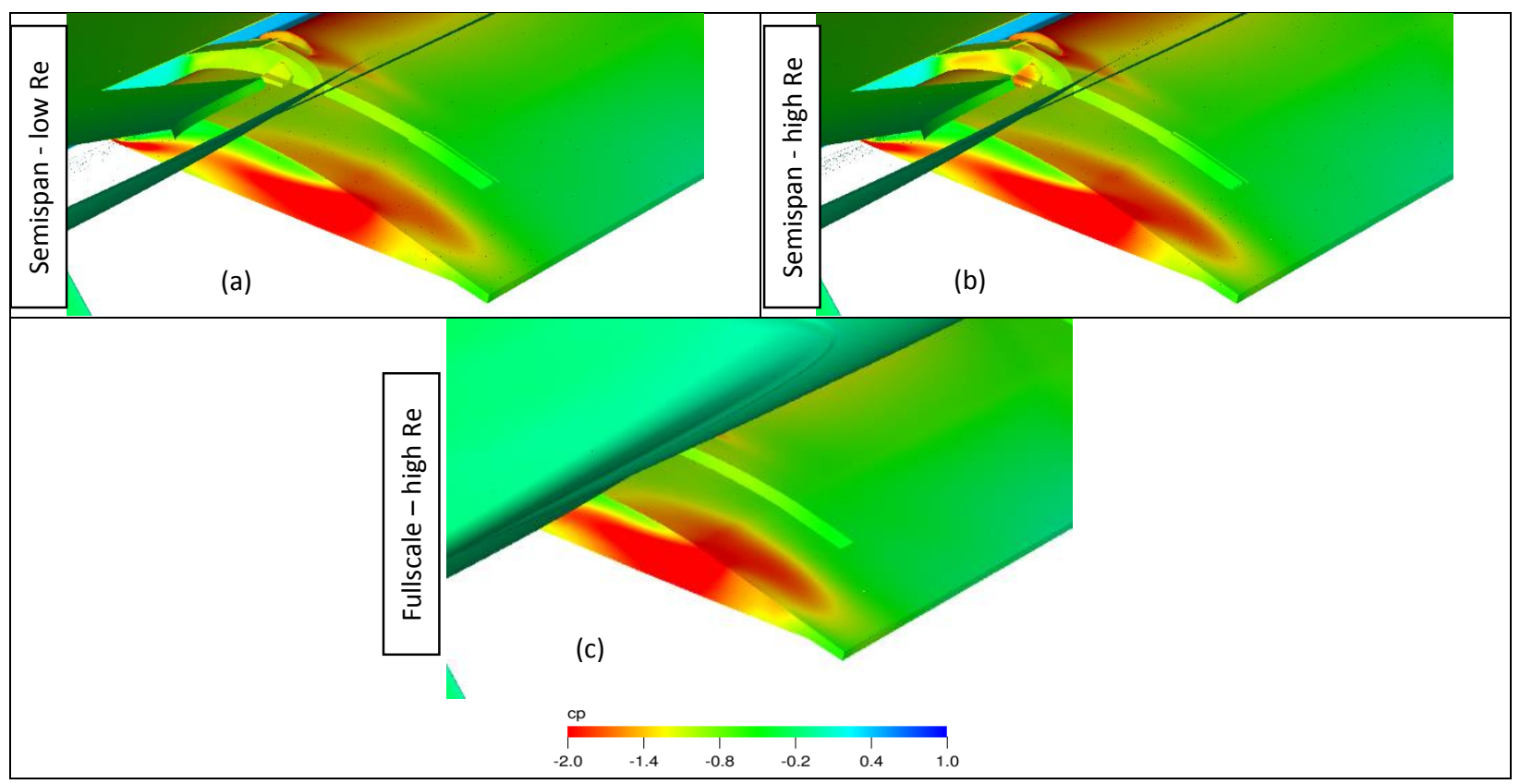

Figure 7. Comparison of static surface pressure distribution at the inboard flap side edge.

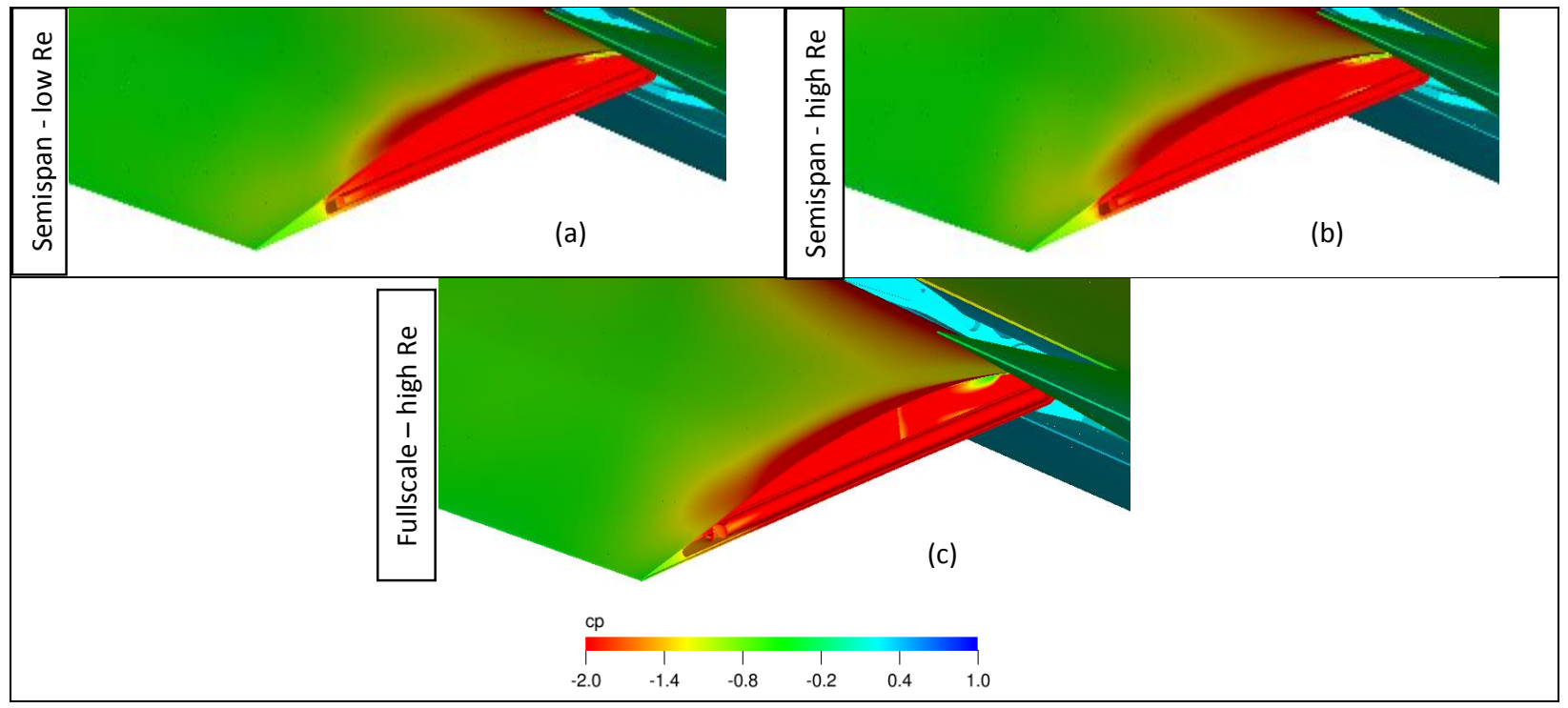

Figure 8. Comparison of static surface pressure distribution at the outboard flap side edge.

For the model scale geometry, the resulting global aerodynamic lift increased by $\sim 3 \%$ with the higher Re while the drag difference between the two configurations remained less than $0.3 \%$ with the higher Re simulation producing the lower value. The differences in lift are spread over most of the wing and flap, as seen in Fig. 9 for the spanwise lift distribution and in Fig. 10 for the pressure coefficient distribution at various sections of the wing and flap. Only the pressure row at the outboard-tip edge (Fig. 10d) shows noticeable changes. The differences between the two 18\% scale, semi-span simulations are caused entirely by Re effects, which result in a stronger vortex on the flap top surface near the leading edge. The stronger vortex provides a more pronounced local pressure suction that is manifested as the second peak (from the flap leading edge) in the pressure coefficient plot. 


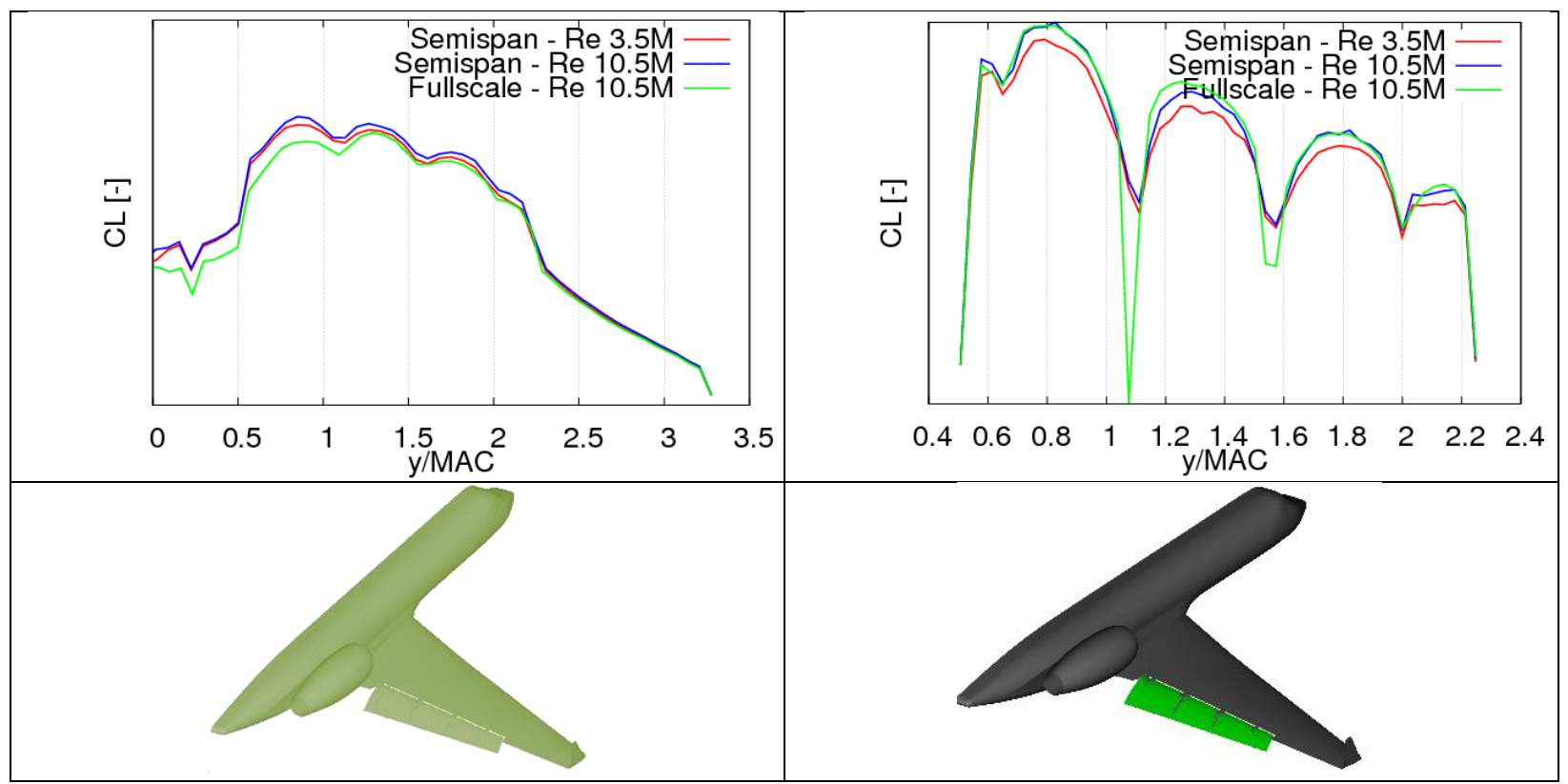

Figure 9. Comparison of the spanwise lift distribution on the half aircraft (left) and flap alone (right).

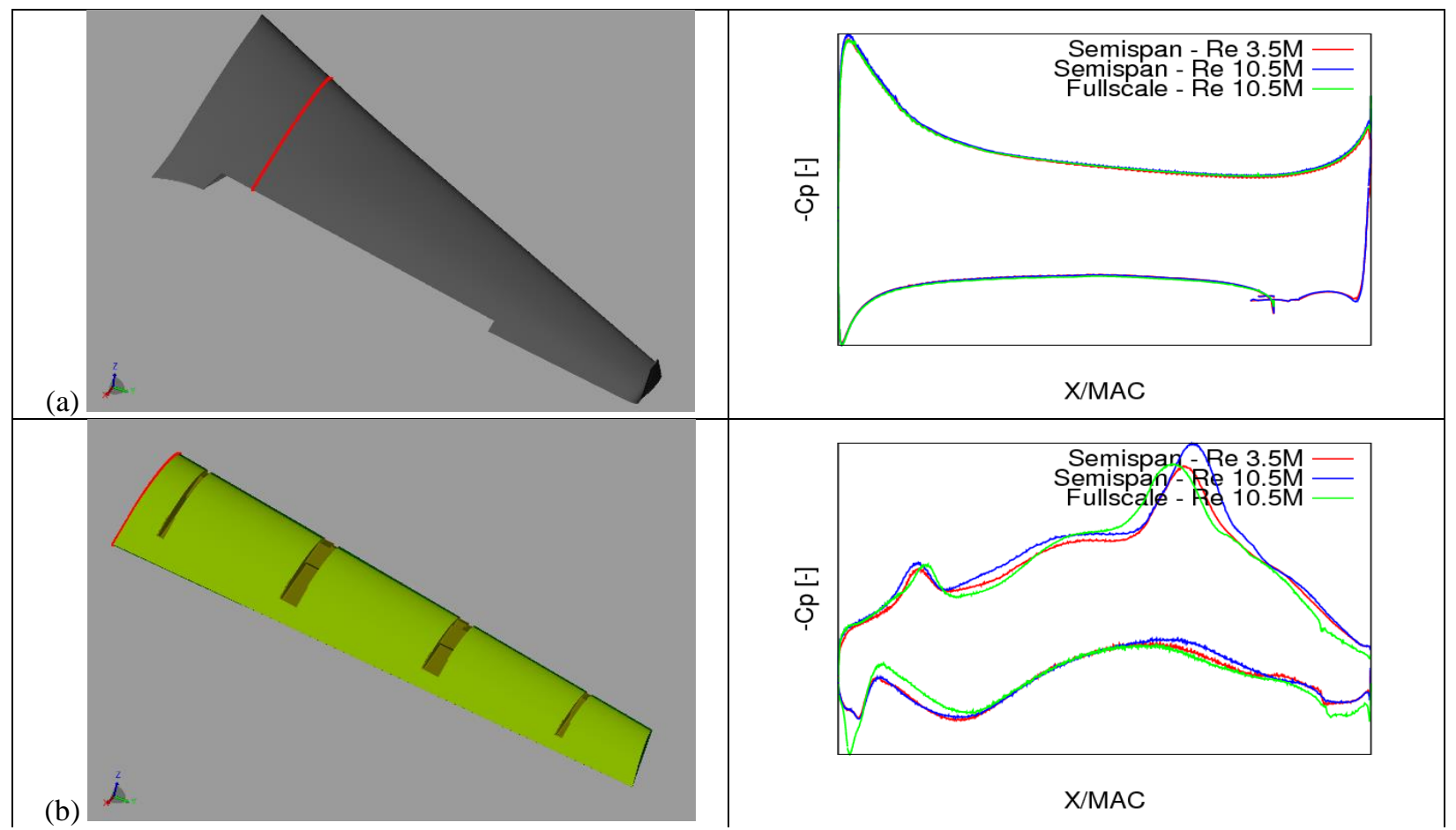

Figure 10. Pressure distribution at various sections on the wing and flap (continued). 


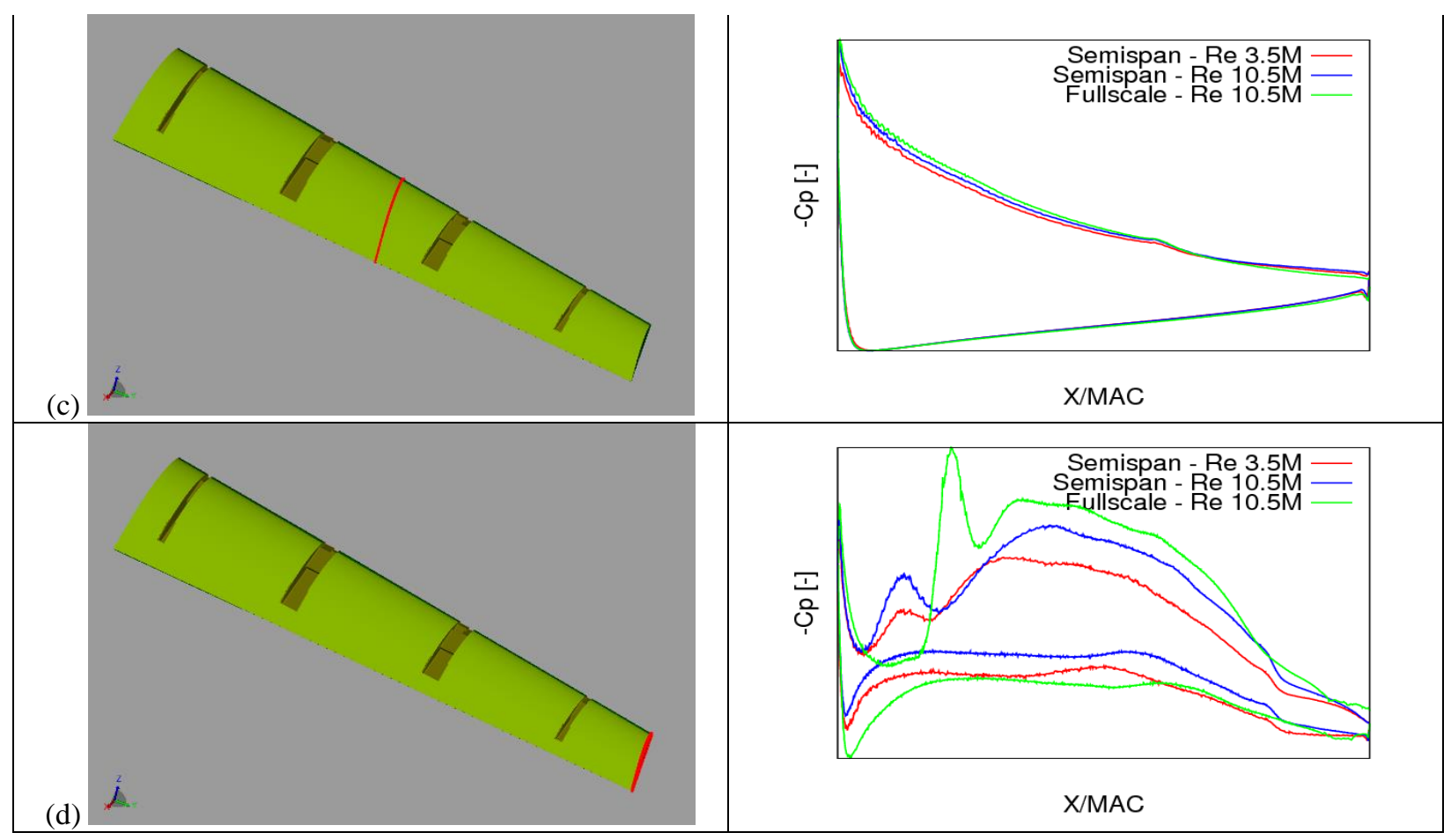

Figure 10. Concluded.

A closer look at a random snapshot of the vortex formation region near the inboard flap tip (Fig. 11a and 11b) shows a high degree of similarity in the larger flow structures between the two Re solutions and qualitative agreement in the resolved smaller structures for the higher Re case. The presence of the finer flow features is attributed to the physical and numerical resolution of the turbulent flow structures at smaller length scales due to the increased Re.

Sample power spectral density (PSD) plots of the fluctuating surface pressures at select locations in the vicinity of the flap inboard and outboard tips corresponding to the position of the physical probes used during $14 \times 22$ wind tunnel measurements ${ }^{7,8,13}$ are shown in Fig. 12. Model scale data have been converted to full-scale for comparison purposes. Surface probes P01 and P06 are located at the inboard tip, while probes P24 and P25 are situated at the outboard tip. In general, at most locations, the spectral shape, frequency content, and magnitude of the fluctuating pressure field are comparable and remain very similar for the two model scale simulations. Nevertheless, at some locations (e.g., probes P24 and P25, Figs. 12c and 12d, respectively) differences emerge. In particular, the presence of new peaks in PSD near the outboard side-edge (Fig. 12c) is mostly driven by the tip cavity that houses a bulb seal, as documented in Ref. 13.

Sample PSD plots for the fluctuating pressure field on the main gear are displayed in Fig. 13. Spectral plots at three measurement probe locations on the gear door and the rear strut are presented. The probe situated on the lower part of the door (Fig. 13a) shows very similar spectra for the two $18 \%$ scale, semi-span model simulations. Large differences are observed for the probe located near the blunt leading edge and the connecting rod of the door (Fig. $13 \mathrm{~b})$. These variations are likely associated with slightly different local flow separation due to Re effects. Overall, the pressure fluctuations on the door are orders of magnitude smaller than those at the flap tips or other components of the MLG, that is, the main gear door is not a prominent noise source for this aircraft and mostly acts as a reflector for impinging acoustic waves generated at other locations. Therefore, the differences in unsteady surface pressure observed at discreet locations have minimal impact on the farfield noise. In contrast, some of the highest pressure fluctuations on the gear are produced at the rear strut near the impingement point for the detached shear layer emanating from the front post. As such, the rear strut is a major contributor to farfield noise. The PSD curves from the two 18\% scale, semi-span model simulations for the probe located on the rear strut (Fig. 13c) are very similar in shape and levels up to the mid-frequency range of $2 \mathrm{kHz}$. As expected, for frequencies above this range, the higher Re simulations yield higher spectral levels due to the finer scale turbulent flow structures being resolved. 


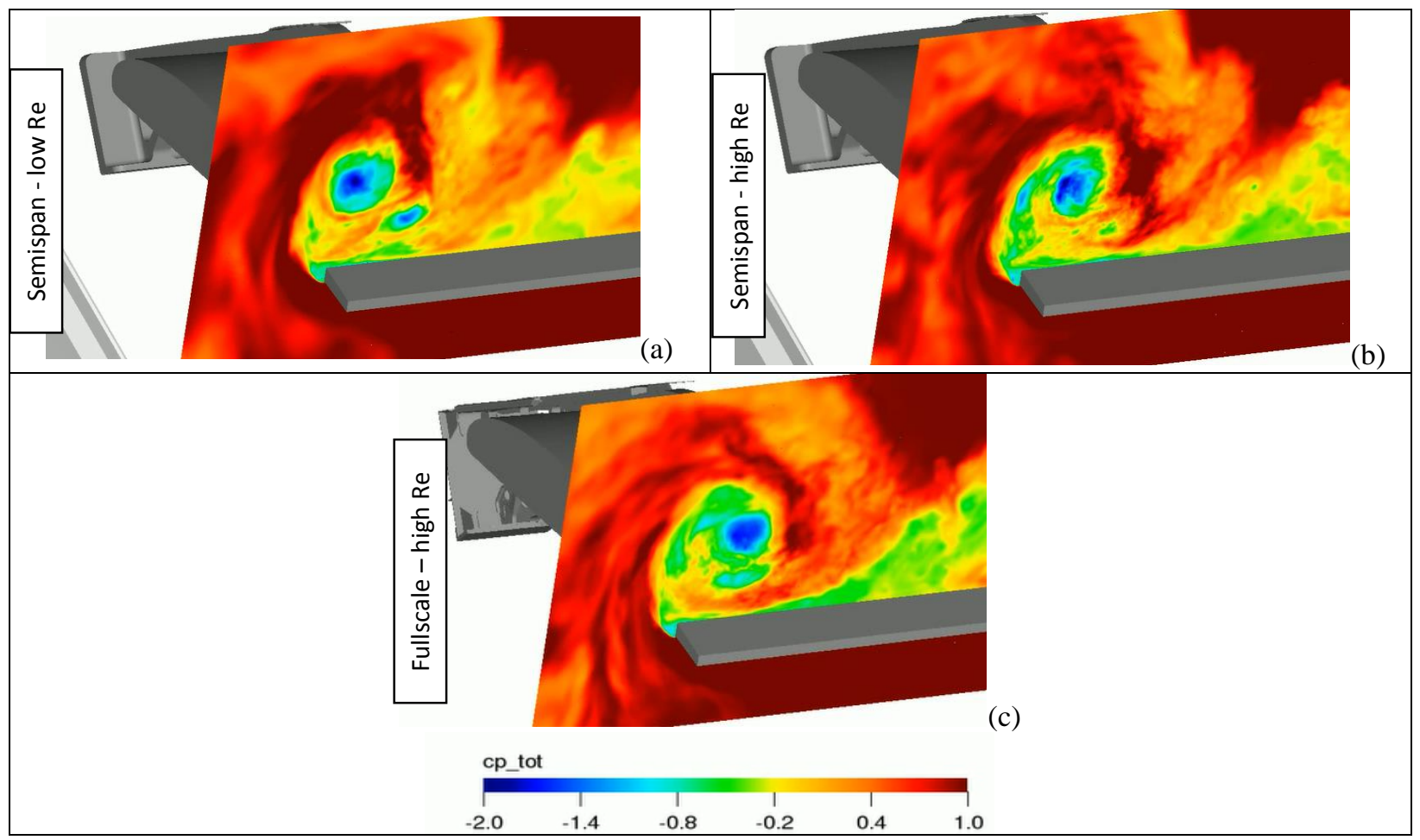

Figure 11. Instantaneous flow structures at the inboard flap.

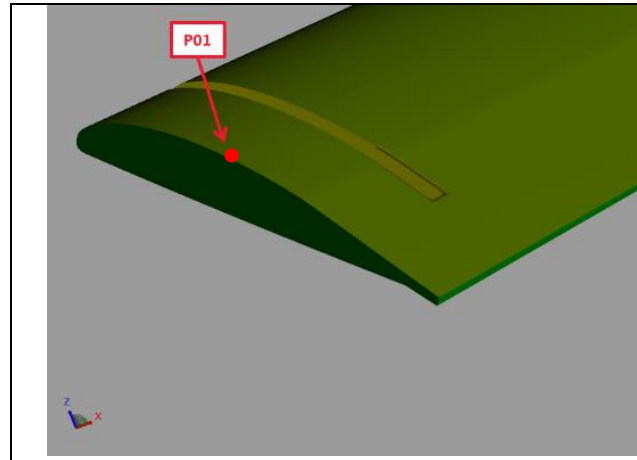

(a)
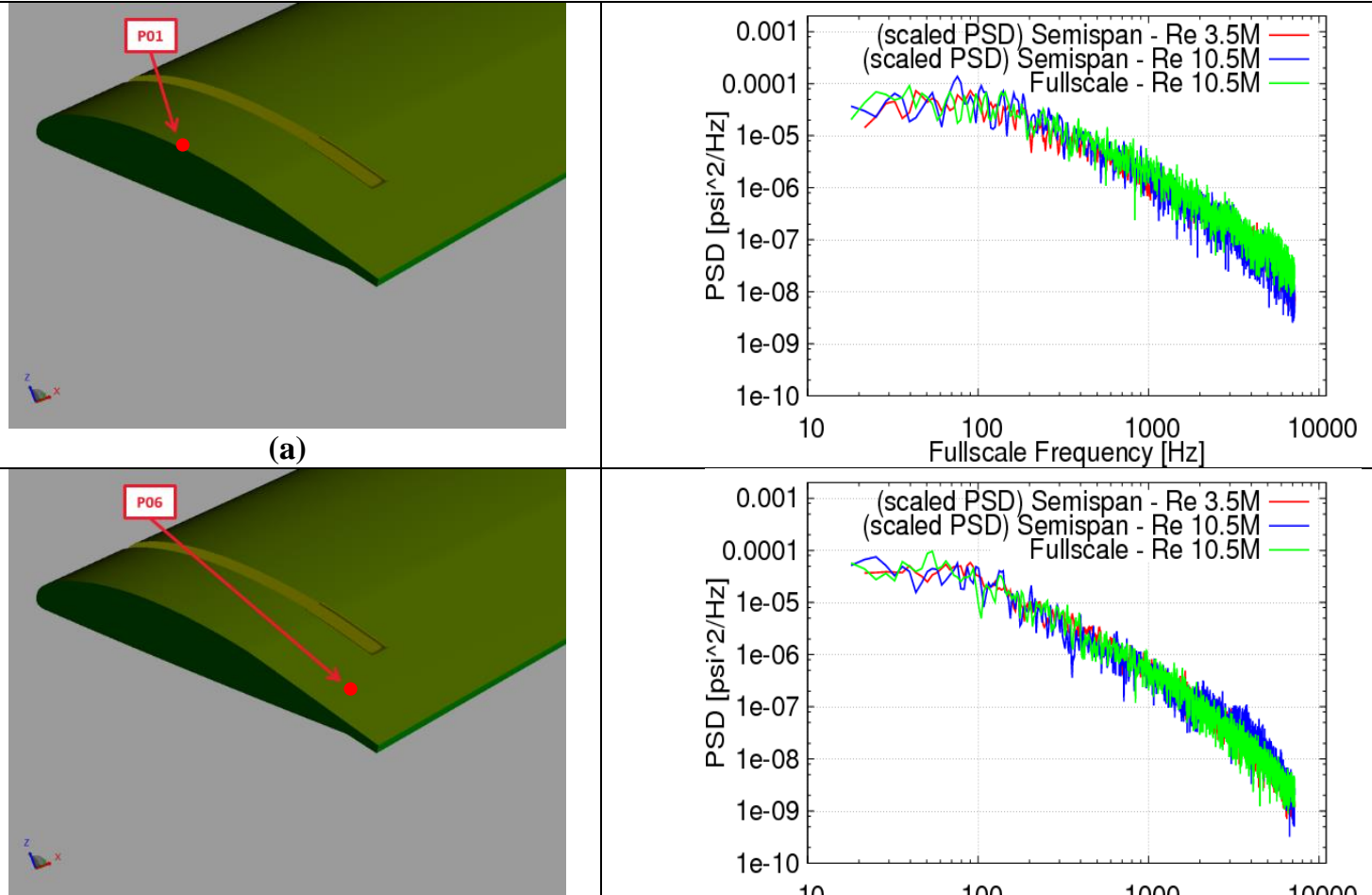

(b)

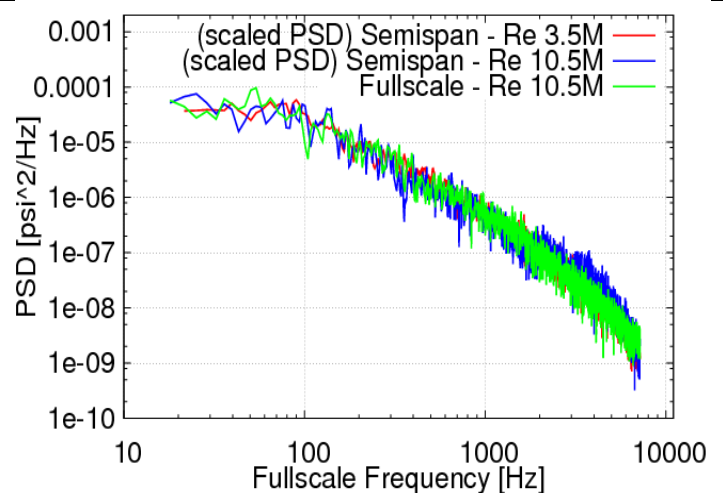

Figure 12. Pressure spectra of surface probes on the flap side edges (continued). 


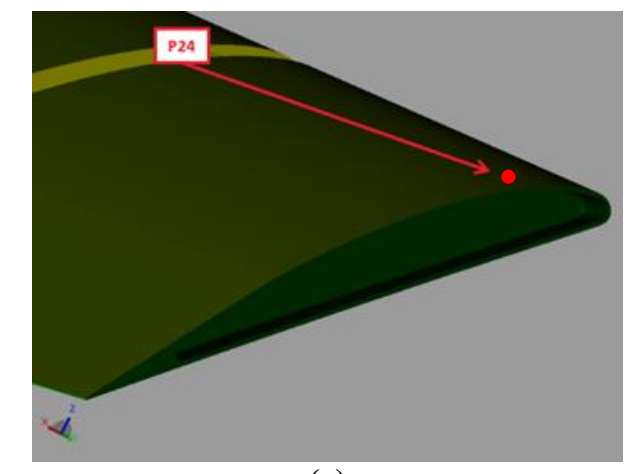

(c)

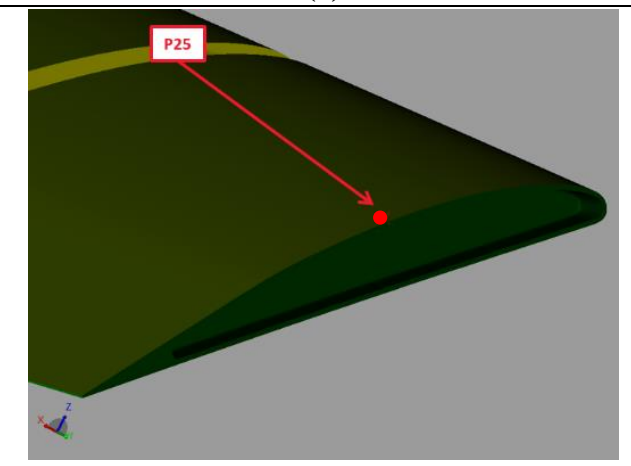

(d)
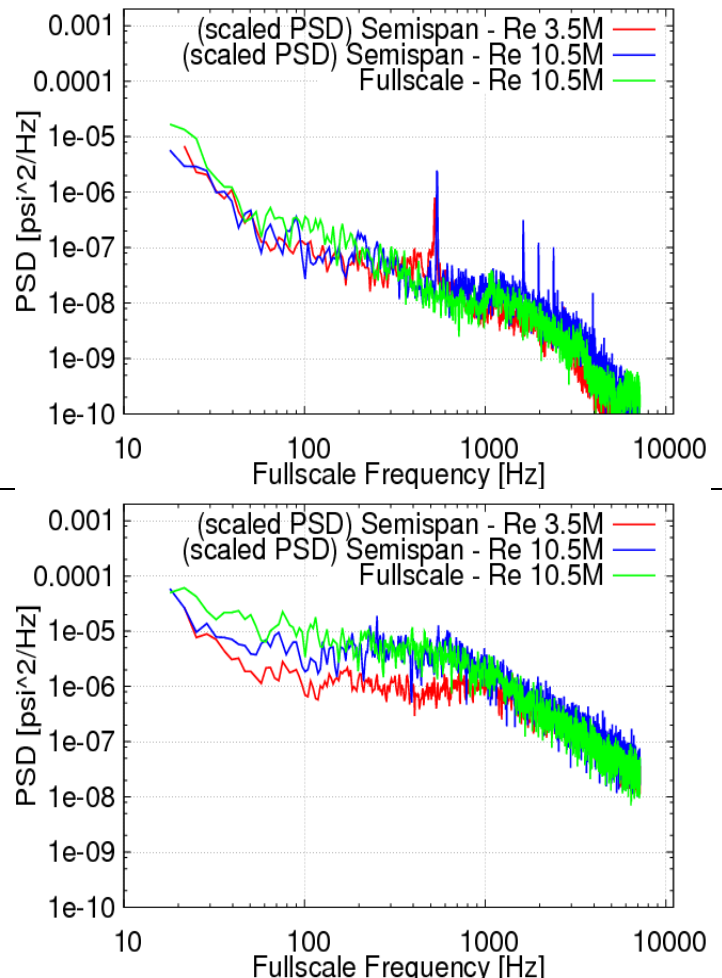

Figure 12. Concluded.

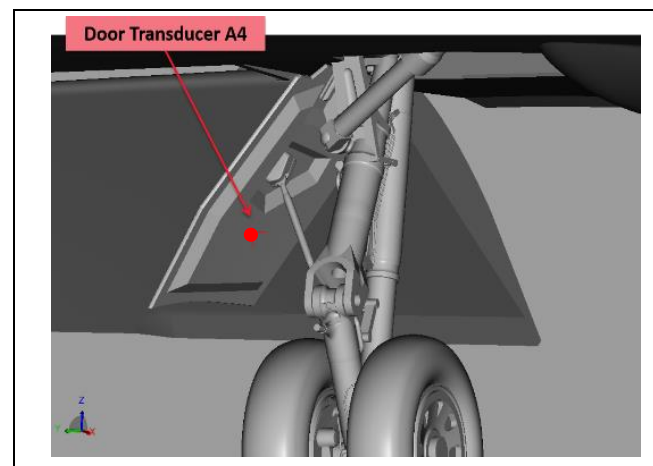

(a)

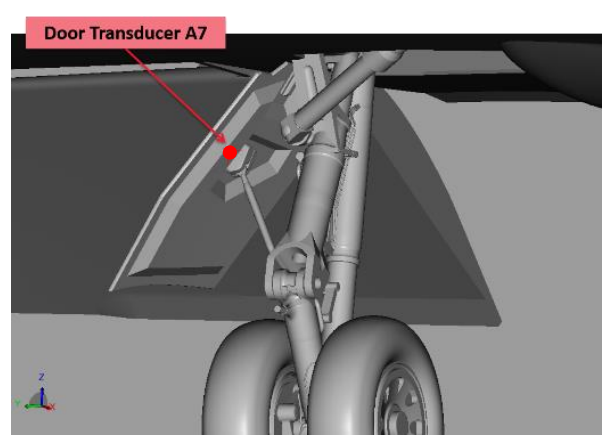

(b)
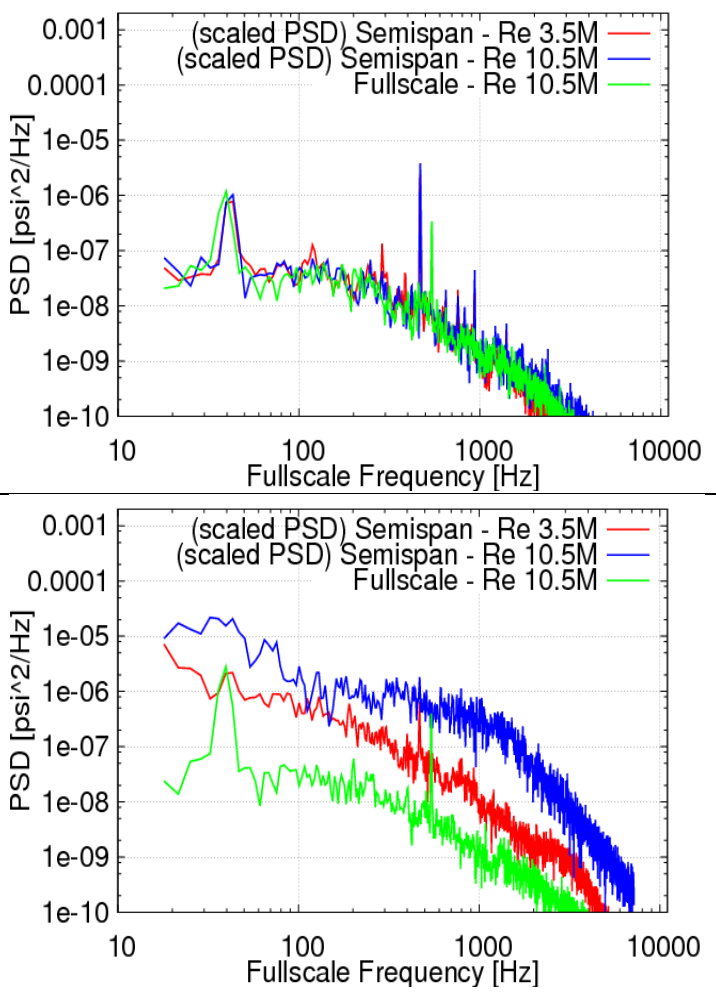

Figure 13. Pressure spectra of surface probes on the MLG geometry (continued). 


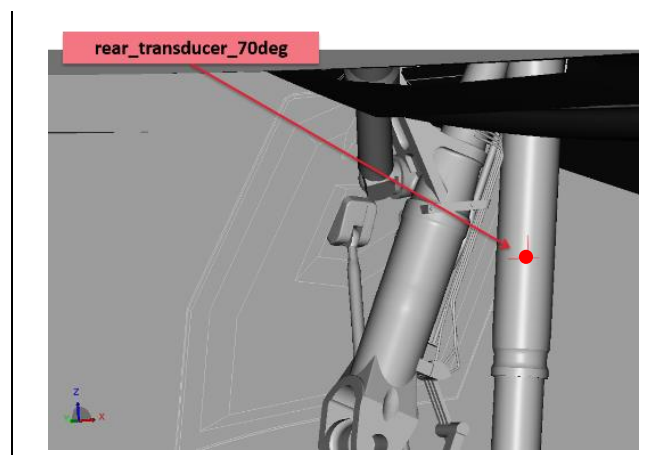

(c)

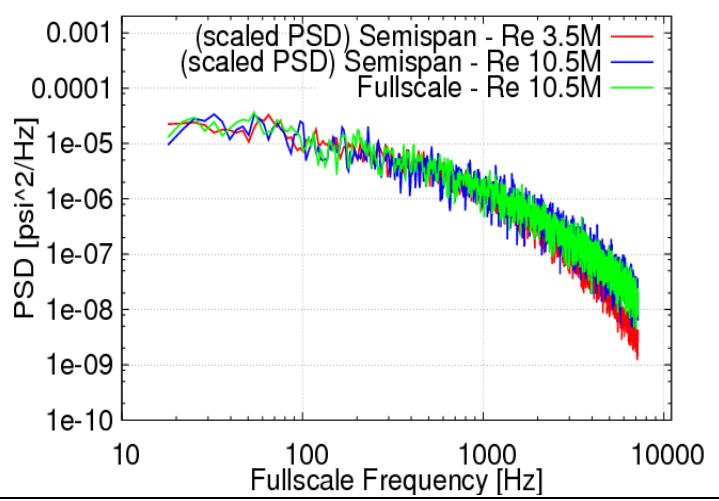

Figure 13. Concluded.

The farfield directivity maps for the $18 \%$ scale, semi-span configuration at the two simulated Re show a similar distribution in terms of directivity pattern and magnitude, as highlighted in Fig. 14. Observe also from the figure that the higher Re (Fig. 14b) produces slightly more noise in the mid-frequency range in the forward direction.

The farfield spectra at an overhead microphone positioned 17.5 feet (at model scale) away from the model are presented in Fig. 15. The spectra for both semi-span simulations are very similar, including the tonal hump associated with the landing gear cavity that occurs at about $200 \mathrm{~Hz}$. In general, other peaks in the mid-frequency range generated by the gear and flap outboard tip cavities are also present in both simulations but are typically more pronounced for the higher Re case. As anticipated, with increased Re, elevated broadband noise levels at high frequencies are observed. The spectral comparison for the two simulations (Fig. 15) confirms that the lower Re sufficiently reproduces the appropriate trends and conditions for airframe noise investigations at the Re encountered during flight testing.

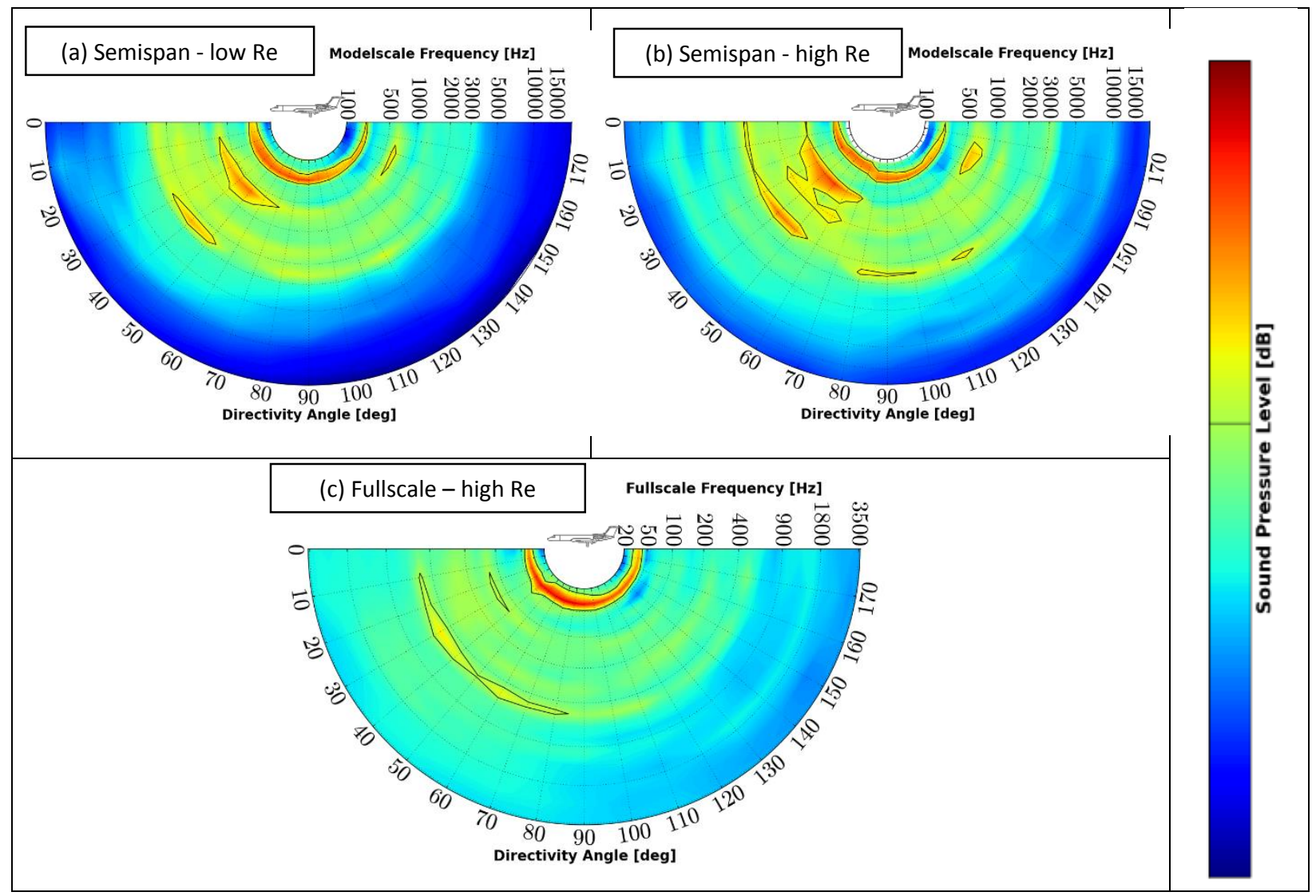

Figure 14. Farfield SPL directivity maps $\left(0^{\circ}\right.$ indicates aircraft forward flight direction). 


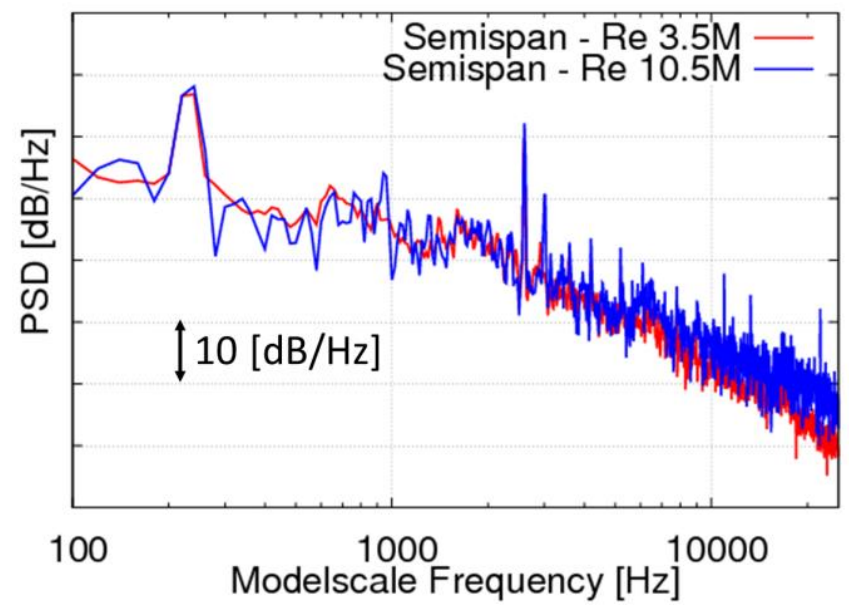

Figure 15. Farfield pressure spectra (overhead position) for the semi-span simulations.

\section{Geometry Effects}

The geometrical effects considered here result from differences in configuration size (18\% vs. $100 \%$ scale), component inclusion (presence of empennage for full-scale configuration), overall physical fidelity, and bracket geometry. For discussion purposes, the first three will be lumped into "form effects." All comparisons are for Re of 10.5 million.

\section{Form Effects}

In general, the flow features and surface pressure patterns from the full-scale configuration are very similar to those obtained from the semi-span simulation, as observed from comparing Figs. 5 through 8 (b and c). Integrated, full-scale global forces are slightly different than those for model scale because of the presence of the tail in the fullscale geometry. For example, lift decreased about $2 \%$ for the full-scale geometry, while drag increased by less than $1 \%$. These trends are opposite to those noted in section IV.B, most likely a result of increased physical fidelity of the full-scale geometry. As shown in Fig. 9, the spanwise lift distribution of the full-scale geometry compares favorably to the semi-span simulation with the larger deviations occurring near the wing root and aft-fuselage region, as well as on the flap, with losses being higher near the more geometrically complex flap brackets. Pressure distributions at various locations on the wing and flap are documented in Fig. 10. The only noticeable differences with model scale pressures occur on the flap. Inspection of the $\mathrm{Cp}$ distribution at the flap inboard edge (Fig. 10b) shows minor differences with the model results. More pronounced changes in flap Cp occur at the outboard edge (Fig. 10d). Here, further decrements in suction side pressure over most of the flap chord are observed. In particular, vortex formation at the edge on the flap top (suction) surface produces a much more prominent but narrower peak downstream of that observed for the $18 \%$ scale model. Although Re effects may play a minor role, most of the observed differences in the outboard tip Cp distribution between full-scale and model scale are due to the more complex cavity and bulb seal assembly of the full-scale geometry. The smaller scale turbulent flow structures captured via the full-scale simulation closely resemble those produced by the mode-scale configuration (see Figs. 11b and 11c).

Unsteady surface pressure spectra for the full-scale and semi-span simulations are displayed in Fig. 12 and Fig. 13. For comparison purposes, the frequencies and levels associated with the semi-span configuration have been converted to full-scale. Generally, the spectra show similar trends at most probe locations. However, at few locations (e.g., probes P25 near the flap outboard tip or Door Transducer A7 close to the leading edge of the gear door), larger variations are observed. Nevertheless, very good overall agreement between model and full-scale computations is seen.

To compare the farfield spectra from the full-scale simulation with the semi-span model results, the corresponding wind tunnel overhead microphone distance has been scaled accordingly. Additionally, only the contribution from the starboard half of the full-scale geometry has been recorded and the model scale spectrum has been scaled for consistency. The full-scale model directivity map shown in Fig. 14c indicates that higher noise levels occur at high frequencies, but generally follows the trends observed for the $18 \%$ scale model in the low frequency range. Farfield noise spectra are presented in Fig. 16. Spectral shape and level, including the low frequency tonal hump associated 
with the gear cavity at $\sim 40 \mathrm{~Hz}$, closely follow the semi-span model trends up to the full-scale frequency of $\sim 1,000 \mathrm{~Hz}$. The full-scale spectrum does not contain the pronounced peaks observed for the semi-span model, but has higher broadband noise levels at high frequencies starting $\sim 1.5 \mathrm{kHz}$. This behavior is attributed mostly to the self-induced noise from the more complex flap brackets, as will be discussed in the next sub-section.

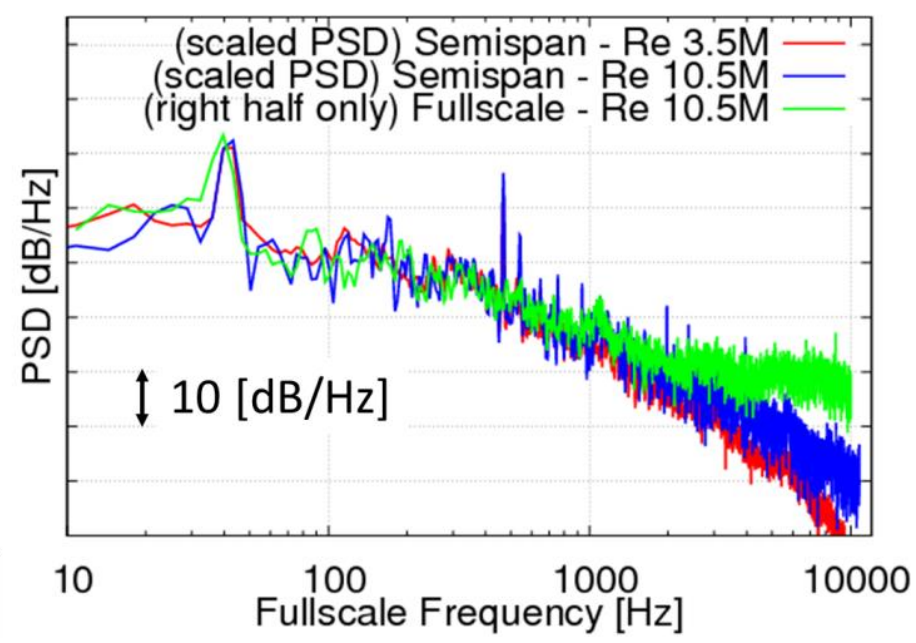

Figure 16. Scaled farfield pressure spectra (overhead position).

\section{Effect of Flap Bracket Geometry on Full-Scale Simulations}

Testing of the $18 \%$ scale semi-span model in the $14 \times 22$ tunnel revealed that the flap brackets could be important secondary noise sources. ${ }^{15}$ Although the model scale brackets were carefully reproduced from full-scale design drawings provided by GAC, fabrication and instrumentation limitations necessitated geometric simplifications and inclusion of wire bundles in the vicinity of the middle two brackets, respectively. The current full-scale, full-aircraft simulations, free of such limitations, corroborated and quantified the true importance of the flap brackets and their ancillary assembly as prominent secondary noise sources contributing significantly to the farfield noise signature at medium and high frequencies. To examine the impact of bracket noise in more detail, two additional configurations were investigated on the full-scale geometry. For the first one, various relatively small gaps and holes in the bracket geometry that were not fully resolved in the original simulation have been closed. Most of these small holes/openings were the result of missing screws, bolts, and other surface definition mismatches in the original full-scale geometry files/descriptions, as partially identified in Fig. 17. This configuration is referred to as "modified brackets." The second configuration is identical to the original baseline, except that all brackets, including the worm gear assembly, have been removed, i.e. the flaps are not connected to the main wing. However, the flap tracks (which house the brackets when the flap is retracted) and vanes were maintained. This configuration is referred to as "no brackets."
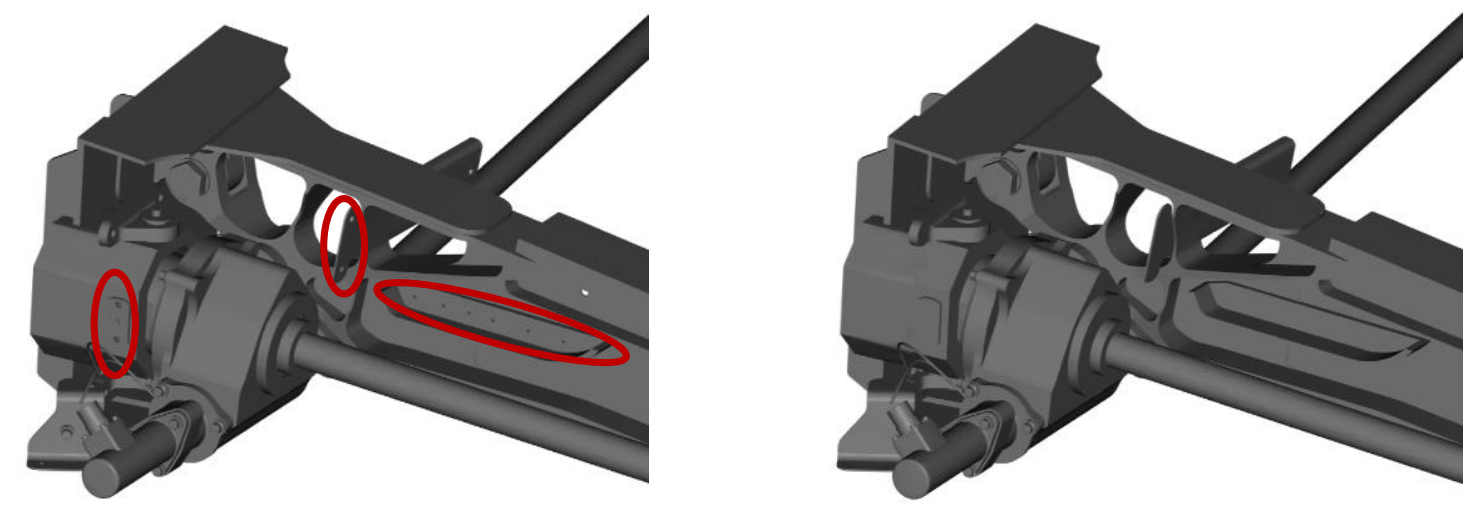

Figure 17. Original (left) and modified (right) brackets, with some of the closed small holes highlighted.

The original and modified bracket configurations produce nearly identical patterns of wake separation in the vicinity of the flap brackets. Removing the brackets completely still leads to flow separation because the bracket tracks 
have been retained. Overall flap loading and formation of the flap side edge vortices remain very similar for all three configurations. As displayed in Figure 18, the differences are mainly confined to the vicinity of the flap track area.

The resulting global lift change is negligible for the modified bracket configuration, whereas an increase of $0.3 \%$ is obtained when the brackets are removed completely. Observe from the flap lift distributions of Figure 19 that the additional lift is mostly gained in the locations of the removed brackets, where flow separation has been reduced.

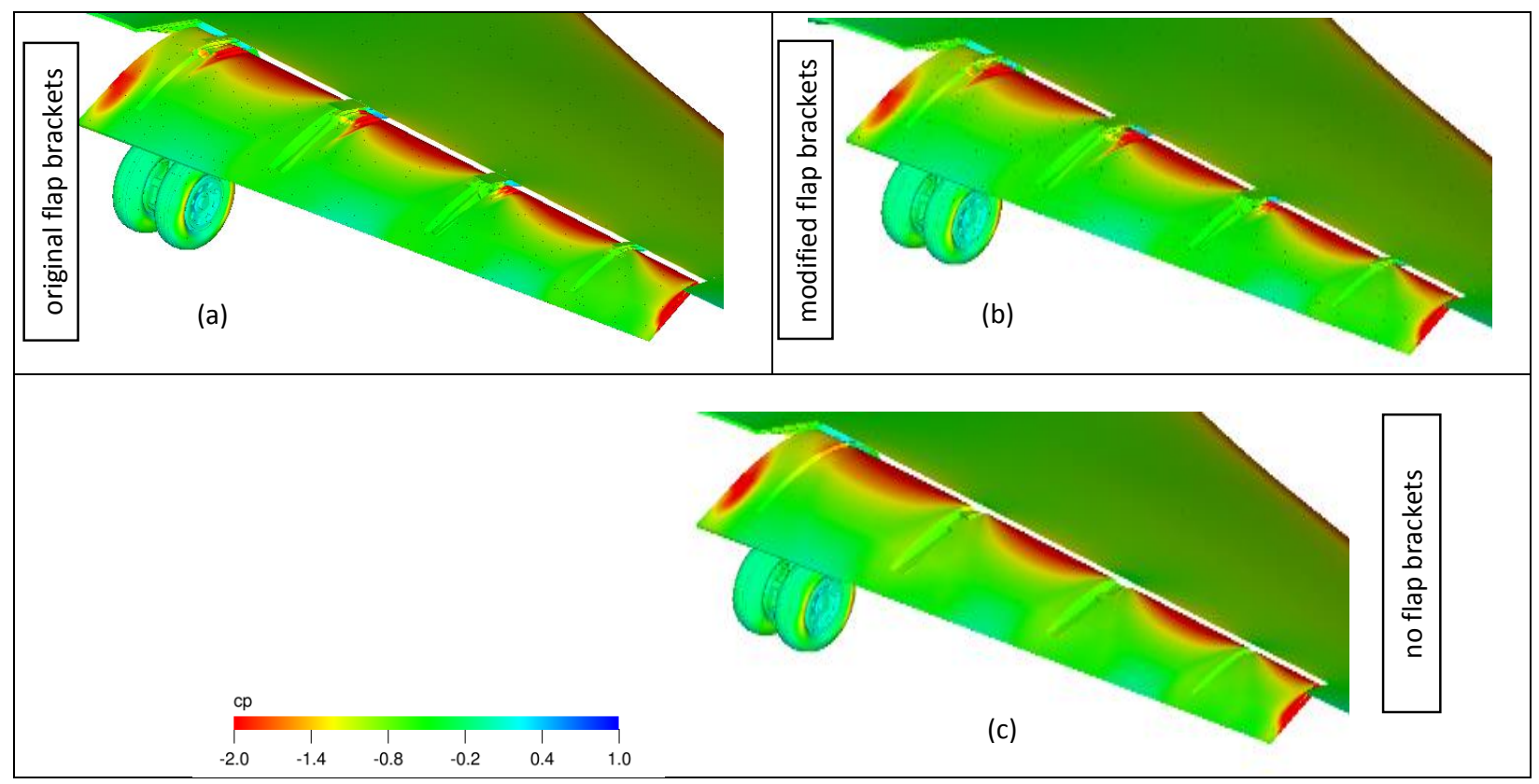

Figure 18. Comparison of static pressure distribution on the flap.

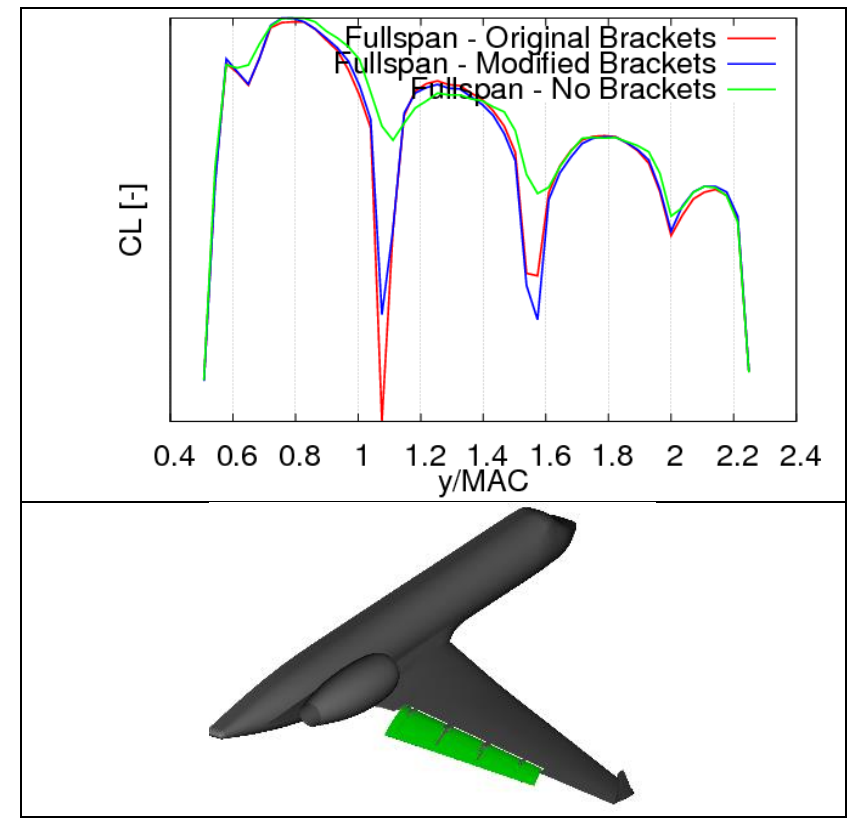

Figure 19. Comparison of the spanwise lift distribution on the flap.

Pressure distribution on the wing was not affected by the modifications nor removal of the flap brackets. Cp distribution on the flap at three spanwise locations is plotted in Fig. 20. The mid-span row (Fig. 20c) depicts a pressure distribution that is nearly the same for all bracket configurations. In contrast, removal of the brackets causes noticeable 
alteration of the steady pressure field at the two primary noise producing regions adjacent to the inboard (Fig. 20b) and outboard (Fig. 20d) side edges. Nevertheless, the alterations can be considered to be moderate, with the pressure distributions at either tip maintaining their overall character relative to the original bracket configuration.

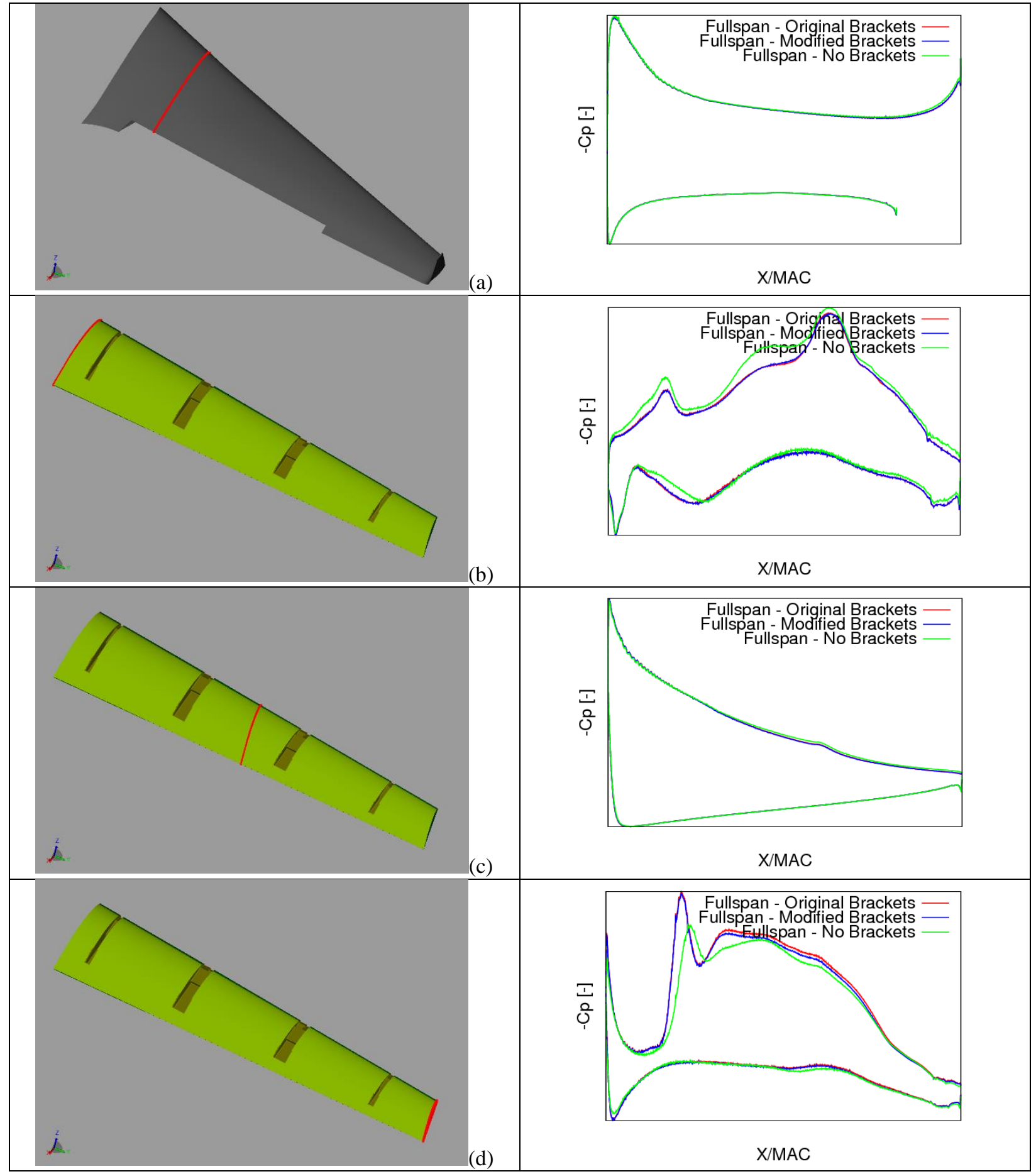

Figure 20. Pressure distribution at various sections on full-scale wing and flap.

PSD plots for probe locations within the flap tip regions are shown in Fig. 21. In general, the unsteady pressure field in the vicinity of the side edges remains unaltered for the modified bracket case, but reveals modest changes in the spectral levels (mostly at higher frequencies) at some of the surface probe locations for the no brackets case. The 
pressure fluctuations on the main landing gear remain nearly the same for the three bracket configurations and therefore, are not presented here.

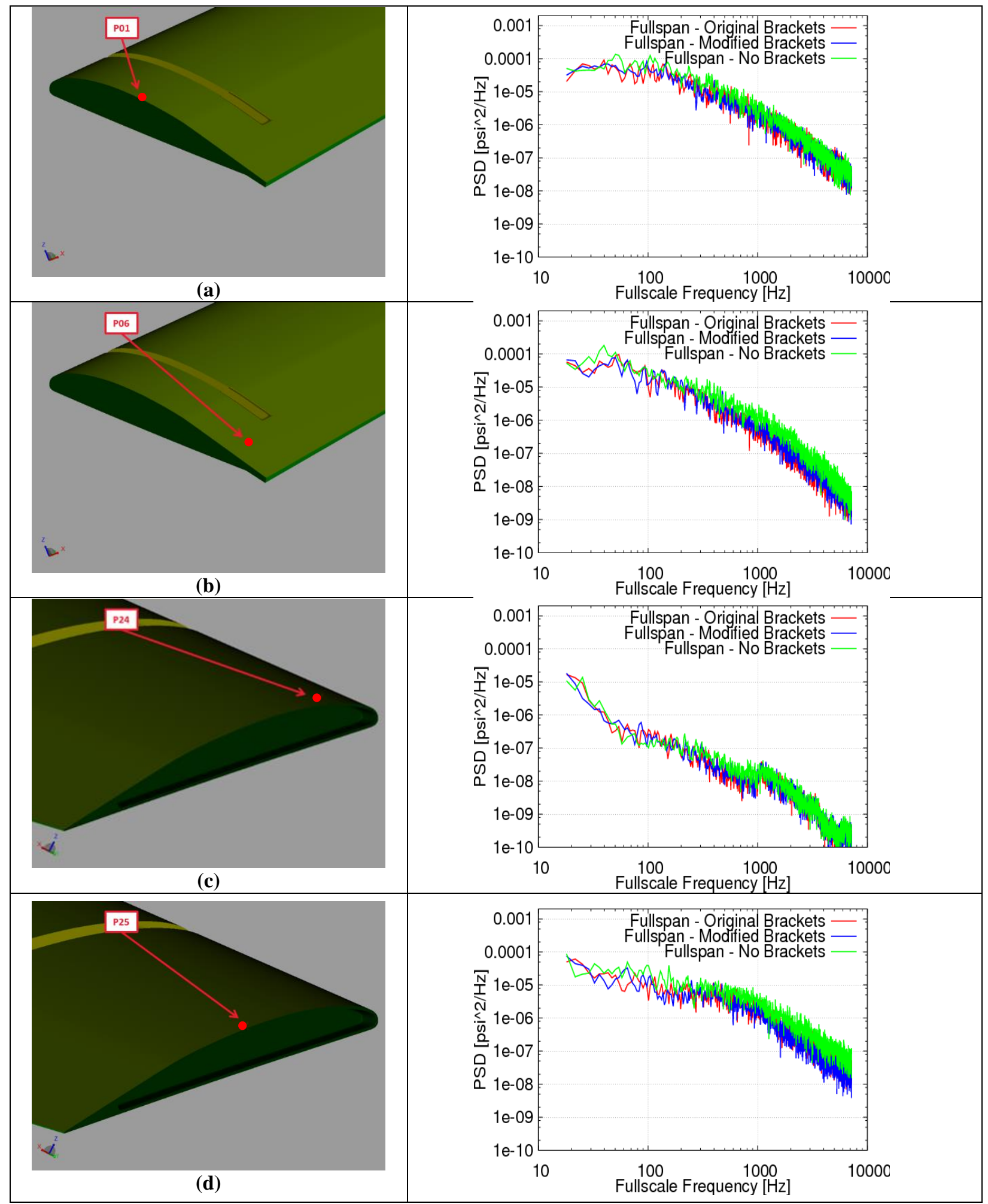

Figure 21. Pressure spectra of surface probes on the flap side edges. 
Figure 22 shows farfield SPL directivity maps for the three bracket configurations on the symmetry plane of the aircraft, at a distance of $29.6 \mathrm{~m}$ and a frequency range between 20 and 8,000 Hz. A large omni-directional peak at very low frequencies, which is due to the landing gear cavity, can be seen in all directivity maps. In general, only minute differences are observed up to $2,000 \mathrm{~Hz}$. Because of various holes and gaps in the original bracket geometry, artificial high frequency noise is generated. By covering these small openings, the high frequency noise content is noticeably reduced for the modified brackets configuration. Removing the brackets completely leads to an additional reduction of high frequency noise, identifying the brackets as a prominent secondary noise source. Keep in mind, however, that the wing cove on the actual aircraft that houses the flap when retracted is more complex than the one simulated here, as it contains additional hardware such as hydraulic lines, wiring, and other fixtures. The presence of these obstructions could potentially alter the local cove flow, and thus, the aeroacoustic characteristics of the brackets.

Farfield spectra at an overhead microphone positioned $120.7 \mathrm{~m}$ away from the aircraft are shown in Fig. 23. The same position is also used to calculate the overall sound pressure levels (OASPL). Note from the figure that, as expected, the reduction in spectral levels is proportional to the "cleanliness" of the bracket configuration. The decrease in levels starts modestly in the mid-frequency range and becomes increasingly larger at higher frequencies. Since high frequency noise levels are more than $10 \mathrm{~dB}$ below broadband peak values (occurring at $150 \mathrm{~Hz}$ ), the observed level of reduction may seem inconsequential. However, this is not the case as the calculation of perceived noise levels (PNL) and effective perceived noise levels (EPNL) emphasizes the high-frequency content of the spectrum. To highlight this point, we have used a D-weighting (which closely tracks the weighting used for PNL computations) to demonstrate the contribution of the brackets to the farfield OASPL: the modified and no bracket configurations produce noise differences of $2.4 \mathrm{dBD}$ and $2.9 \mathrm{dBD}$, respectively, as compared to the original brackets. These differences became very relevant during evaluation of several airframe noise reduction concepts on the same geometry, ${ }^{37}$ where it was realized that bracket noise masks the full acoustic potential of the flap tip treatments being evaluated. The farfield propagation analysis reported here did not include atmospheric absorption effects.

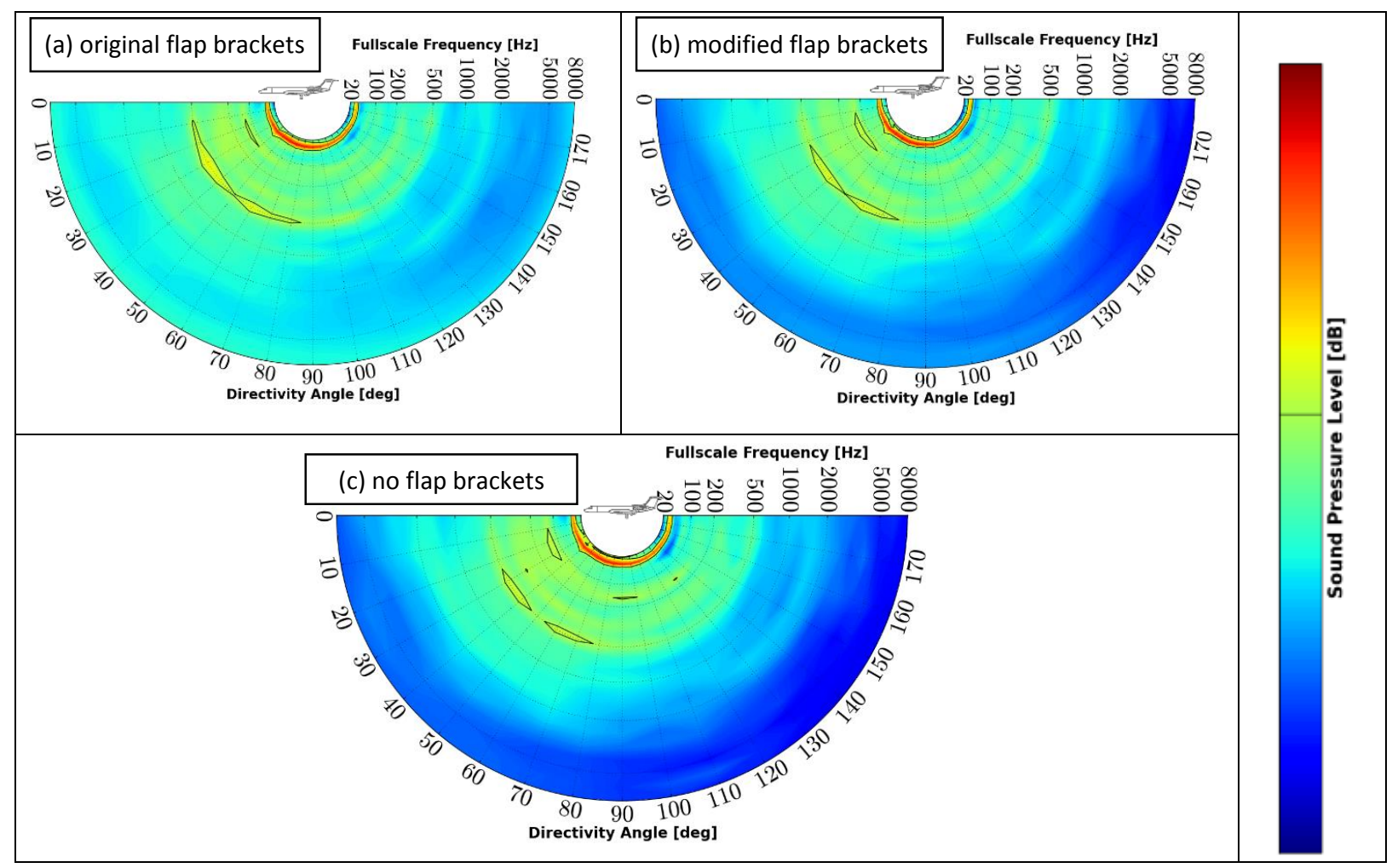

Figure 22. Farfield SPL directivity map for the original brackets $\left(0^{\circ}\right.$ indicate aircraft forward flight direction). 


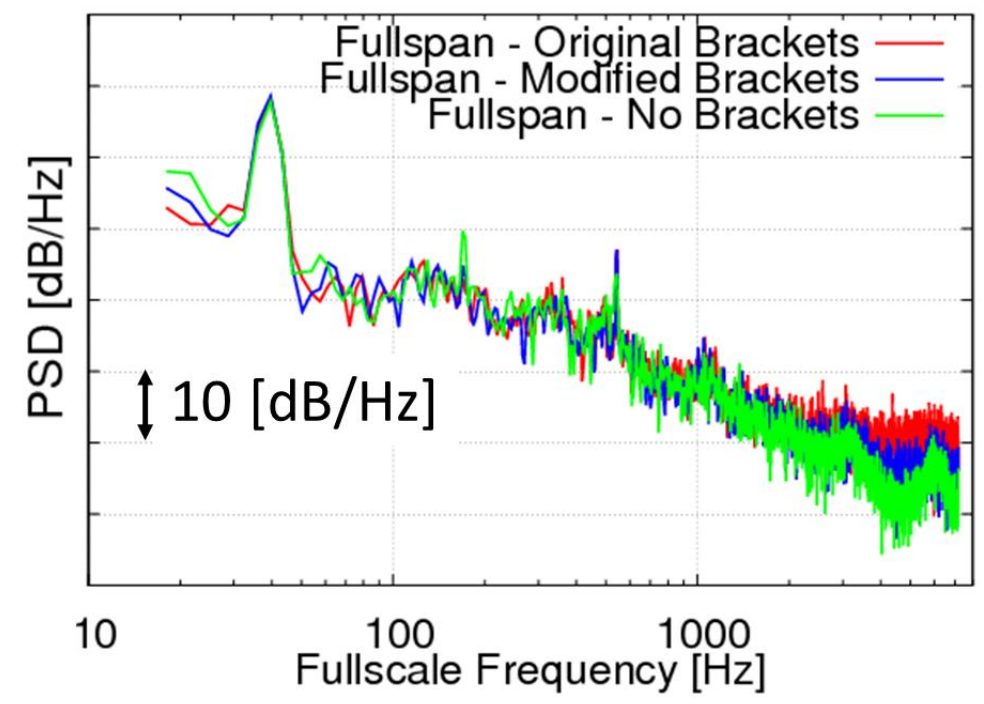

Figure 23. Farfield pressure spectra (flyover microphone position).

\section{Conclusions}

Unsteady flow simulations using the lattice Boltzmann solver PowerFLOW ${ }^{\circledR}$ were used to analyze the aerodynamic and aeroacoustic behavior of high-fidelity Gulfstream aircraft models in landing configuration. The geometries simulated were an $18 \%$ scale semi-span wind tunnel model and a full-scale, full-span version of the model possessing some of the finer geometric details found in the actual aircraft. The $18 \%$ scale model was simulated at Re of 3.4 million and 10.5 million based on the mean aerodynamic chord of the wing, corresponding to wind tunnel conditions and half of the flight Re, respectively. The full-scale aircraft simulations were conducted at $\operatorname{Re}$ of 10.5 million. The simulation results were used to carefully compare and isolate Re and geometry effects. The results indicate that the high fidelity, semi-span model at Re of 3.5 million is able to capture most of the aerodynamic and aeroacoustic effects in terms of both near field surface pressure fluctuations and farfield acoustics. Only small differences were observed, mostly related to high frequency behavior. A dedicated investigation on the effect of fullscale flap bracket geometry was also performed, highlighting the importance of these structures to the high frequency content of the farfield spectra.

\section{Acknowledgments}

This work was supported by the Environmentally Responsible Aviation (ERA) project under the Integrated Aviation Systems Program (IASP) of NASA. The authors would like to thank Jason Appelbaum of Exa Corporation for post-processing a portion of the data presented in this paper. All the simulations were performed on the Pleiades supercomputer at the NASA Advanced Supercomputing (NAS) facility at Ames Research Center. The logistical support provided by NAS staff, in particular Yan-Tyng (Sherry) Chang of Computer Sciences Corporation, is greatly appreciated.

\section{References}

1 Dobrzynski, W., "Almost 40 Years of Airframe Noise Research: What Did We Achieve," J. Aircraft, Vol. 47, No.2, pp. 353-367, 2010.

2 Fink, M., "Noise Component Method for Airframe Noise," J. Aircraft, Vol. 16, No. 10, pp. 659-665, 1979.

3 Guo, Y., Yamamoto, K .J., and Stoker, R. W., "Component-Based Empirical Model for High-Lift System Noise Prediction," J. Aircraft, Vol. 40, No. 5, pp. 914-922, 2003.

4 Rackl, R. G., Miller, G., Guo, Y., and Yamamoto, K., "Airframe Noise Studies - Review and Future Directions," NASA Technical Report NASA/CR-2005-213767, June 2005.

5 https://info.aiaa.org/tac/ASG/FDTC/DG/BECAN_files_/BANCIII.htm, visited on $16^{\text {th }}$ October 2014. 
6 Lockard, D. "Summary of the Tandem Cylinder Solutions from the Benchmark problems for Airframe Noise Computations-I Workshop," AIAA Paper 2011-0353, 2011.

7 Khorrami, M. R. and Mineck, R. E., "Towards Full Aircraft Airframe Noise Prediction: Detached Eddy Simulations," AIAA Paper 2014-2480, 2014.

8 Khorrami, M. R., Fares, E., and Casalino, D., "Towards Full-Aircraft Airframe Noise Prediction: Lattice-Boltzmann Simulations," AIAA Paper 2014-2481, 2014.

9 Casalino, D., Hazir, A., Fares, E., Duda, B., and Khorrami, M., "On the Connection between Flap Side-Edge Noise and Tip Vortex Dynamics," AIAA Paper 2015-2992, 2015.

${ }^{10}$ Khorrami, M., Mineck, R., Yao, C., and Jenkins, N., "A Comparative Study of Simulated and Measured Gear-Flap Flow Interaction," AIAA Paper 2015-2989, 2015.

${ }^{11}$ Fares, E., Casalino, D., and Khorrami, M., "Evaluation of Airframe Noise Reduction Concepts via Simulations Using a Lattice Boltzmann Approach,” AIAA Paper 2015-2988, 2015.

${ }^{12}$ Khorrami, M. R., Hannon, J. A., Neuhart, D. H., Markowski, G. A., and Van de Ven, T., "Aeroacoustic Studies of a High-Fidelity Aircraft Model: Part 1-Steady Aerodynamic Measurements," AIAA Paper 2012-2233, 2012.

${ }^{13}$ Khorrami, M. R. and Neuhart, D. H, "Aeroacoustic Studies of a High-Fidelity Aircraft Model: Part 2- Unsteady Surface Pressures," AIAA Paper 2012-2234, 2012.

${ }^{14}$ Neuhart, D., Hannon, J., and Khorrami, M. R., “Aerodynamic Measurements of a Gulfstream Aircraft Model with and without Noise Reduction Concepts," AIAA Paper 2014-2477, 2014.

${ }^{15}$ Khorrami, M. R., Humphreys, W. M. Jr., Lockard, D. P., and Ravetta, P. A., "Aeroacoustic Evaluaion of Flap and Landing Gear Reduction Concepts," AIAA Paper 2014-2478, 2014.

${ }^{16}$ Chen, H., "Volumetric Formulation of the Lattice-Boltzmann Method for Fluid Dynamics: Basic Concept," Physical Review E, Vol. 58, No. 3, pp. 3955-3963, 1998.

${ }^{17}$ Chen, H., Texeira, C., and Molvig, K., "Realization of Fluid Boundary Condition via Discrete Boltzmann Dynamics," Int. Journal of Modern Physics C, Vol. 09, pp. 1281-1292, 1998.

${ }^{18}$ Yakhot, V. and Orszag, S. A., "Renormalization Group Analysis of Turbulence. I. Basic Theory," J. Sci. Comput., Vol. 1, No. 2, pp. 3-51, 1986.

${ }^{19}$ Chen, H., Kandasamy, S., Orszag, S., Shock, R., Succi, S., and Yakhot, V., "Extended Boltzmann Kinetic Equation for Turbulent Flows," Science, No. 301, pp. 633-636, 2003.

${ }^{20}$ Chen, S., and Doolen, G. D., "Lattice Boltzmann Method for Fluid Flows," Annual Review of Fluid Mechanics, Vol. 30, pp 329-364, 1998.

${ }^{21}$ Fares, E. and Noelting, S., "Unsteady Flow Simulation of a High-Lift Configuration using a Lattice-Boltzmann Approach," AIAA Paper 2011-0869, 2011.

${ }^{22}$ Casalino, D., Noelting, S., Fares, E., Van de Ven, T., Perot, F., and Bres, G., "Towards Numerical Aircraft Noise Certification: Analysis of a Full scale Landing Gear in Fly-Over Configuration," AIAA Paper 2012-2235, 2012.

${ }^{23}$ Casalino D., Ribeiro, A. F., Fares, E., Nölting, S., Mann, A., Perot, F., Li, Y., Lew, P., Sun, C., Gopalakrishnan, P., Zhang, R., Chen, H., and Habibi, K., " "Towards Lattice Boltzmann Predictions of Turbofan Noise," AIAA Paper 2014-3101, 2014.

${ }^{24}$ Chen, H., Chen, S., and Matthaeus, W., "Recovery of the Navier-Stokes Equations Using a Lattice-Gas Boltzmann Method," Physical Review A, Vol. 45, No. 8, pp. 5339-5342, 1992.

${ }^{25}$ Qian Y. H., D'Humières, D., and Lallemand, P., "Lattice BGK Models for Navier-Stokes Equation," Europhysics Letters, Vol. 17, pp. 479-484, 1992.

${ }^{26}$ Marié, S., Ricot, D., and Sagaut, P., "Comparison between lattice Boltzmann method and Navier-Stokes high order schemes for computational aeroacoustics," J. of Computational Physics, Vol. 228, pp. 1056-1070, 2009.

${ }^{27}$ Brès, G. A., Pérot, F., and Freed, D. "Properties of the Lattice-Boltzmann Method for Acoustics," AIAA Paper 2009-3395, May 2009.

${ }^{28}$ Shan, X.,Yuan, X. F., and Chen, H., "Kinetic theory representation of hydrodynamics: a way beyond the NavierStokes equation," J. of Fluid Mechanics, Vol. 550, pp 413- 441, 2006.

${ }^{29}$ Zhang, R., Shan, X., and Chen, H., "Efficient kinetic method for fluid simulation beyond the Navier-Stokes equation," Physical Review E, Vol. 74, p. 046703, 2006.

${ }^{30}$ Koenig, B, and Fares, E., "Validation of a Transonic Lattice-Boltzmann Method for the NASA Common Research model," AIAA Paper 2016-2023, 2016. 
${ }^{31}$ Chen, H., Orszag, S., Staroselsky, I., and Succi, S. "Expanded Analogy between Boltzmann Kinetic Theory of Fluid and Turbulence," J. of Fluid Mechanics, Vol. 519, pp. 307-314, 2004.

32 Fares, E. "Unsteady Flow Simulation of the Ahmed Reference Body using a Lattice Boltzmann Approach," J. of Computers and Fluids, No. 35, pp. 940-950, 2006.

33 Ffowcs Williams, J. E. and Hawkings, D. L., "Sound Generated by Turbulence and Surfaces in Arbitrary Motion," Philosophical Transactions of the Royal Society, Vol. A264, No. 1151, pp. 321-342, 1969.

${ }^{34}$ Farassat, F. and Succi, G. P., “The Prediction of Helicopter Discrete Frequency Noise," Vertica, Vol. 7, No. 4, pp. 309-320, 1983.

35 Najafi-Yazdi, A., Brès, G. A., and Mongeau, L., “An Acoustic Analogy Formulation for Moving Sources in Uniformly Moving Media,” Proceeding of The Royal Society of London A, Vol. 467 (2125), pp. 144-165, 2011.

${ }^{36}$ Khorrami, M. R., Lockard, D. P., Humphreys, Jr., W. M., Choudhari, M. M., and Van de Ven, T., "Preliminary Analysis of Acoustic Measurements from the NASA-Gulfstream Airframe Noise Flight Test," AIAA Paper 20082814, May 2008.

${ }^{37}$ Khorrami, M. R., Duda, B., Hazir, A., and Fares, E., "Computational Evaluation of Airframe Noise Reduction Concepts at Full Scale," Paper to be presented at the $22^{\text {nd }}$ AIAA/CEAS Aeroacoustics Conference in Lyon, France, May-June 2016. 